\title{
RNF2 is recruited by WASH to ubiquitinate AMBRA1 leading to downregulation of autophagy
}

\author{
Pengyan $\mathrm{Xia}^{1,2,{ }^{1,} \text {, Shuo Wang }}{ }^{1, *}$, Guanling Huang ${ }^{1,2}$, Ying $\mathrm{Du}^{1}$, Pingping $\mathrm{Zhu}^{1}$, Man $\mathrm{Li}^{1,2}$, Zusen Fan ${ }^{1}$ \\ ${ }^{I}$ Key Laboratory of Infection and Immunity of CAS, Institute of Biophysics, Chinese Academy of Sciences, Beijing 100101, China; \\ ${ }^{2}$ University of Chinese Academy of Sciences, Beijing 100049, China
}

\begin{abstract}
WASH (Wiskott-Aldrich syndrome protein (WASP) and SCAR homolog) was identified to function in endosomal sorting via Arp2/3 activation. We previously demonstrated that WASH is a new interactor of BECN1 and present in the BECN1-PIK3C3 complex with AMBRA1. The AMBRA1-DDB1-CUL4A complex is an E3 ligase for K63-linked ubiquitination of BECN1, which is required for starvation-induced autophagy. WASH suppresses autophagy by inhibition of BECN1 ubiquitination. However, how AMBRA1 is regulated during autophagy remains elusive. Here, we found that RNF2 associates with AMBRA1 to act as an E3 ligase to ubiquitinate AMBRA1 via K48 linkage. RNF2 mediates ubiquitination of AMBRA1 at lysine 45. Notably, RNF2 deficiency enhances autophagy induction. Upon autophagy induction, RNF2 potentiates AMBRA1 degradation with the help of WASH. WASH deficiency impairs the association of RNF2 with AMBRA1 to impede AMBRA1 degradation. Our findings reveal another novel layer of regulation of autophagy through WASH recruitment of RNF2 for AMBRA1 degradation leading to downregulation of autophagy.
\end{abstract}

Keywords: WASH; RNF2; AMBRA1; polyubiquitination; the BECN1-PIK3C3 complex

Cell Research (2014) 24:943-958. doi:10.1038/cr.2014.85; published online 1 July 2014

\section{Introduction}

Autophagy is an essential process to degrade cellular proteins and organelles for cell survival under nutrient-limited conditions or to recycle old cellular components for cellular renovation [1-4]. Upon receiving outside stimuli such as amino acid or growth factor withdrawal, double-membrane structures are formed from either the endoplasmic reticulum (ER) or the mitochondrial membrane to enclose intracellular protein aggregates or damaged organelles [5-9], which is referred to as autophagosomes. The autophagosome merges with the lysosome to form an autolysosome in which the target materials are degraded [10]. Autophagosome formation is mainly regulated by the $\mathrm{BECN} 1-\mathrm{PIK} 3 \mathrm{C} 3$ (also known

\footnotetext{
*These two authors contributed equally to this work.

Correspondence: Zusen Fan

Tel: 86-10-64888457; Fax: 86-10-64871293

E-mail: fanz@moon.ibp.ac.cn

Received 12 March 2014; revised 1 May 2014; accepted 26 May 2014; published online 1 July 2014
}

as Beclin 1-Vps34) complex at the early stage of autophagy $[11,12]$. Recently, several major autophagy regulators were identified in vivo in the large BECN1-PIK3C3 complex, including ATG14 (also known as ATG14L, or Barkor), UVRAG (UV irradiation resistance-associated gene), AMBRA1, and Rubicon (RUN domain and cysteine-rich domain containing, BECN1-interacting protein) [13-16]. Intriguingly, the stability of the BECN1PIK3C3 complex is codependent on each component [14], suggesting that each component of this complex plays a critical role in the modulation of autophagy.

Among these components, BECN1 (ortholog of yeast Atg6) plays an important role in autophagosome formation and maturation [14-17]. BECN1 associates with PIK3C3 to activate its kinase activity, phosphorylating the D-3 position of the inositol ring of phosphatidylinositol to generate PI3P, which is required for the formation of the autophagosome structure [18-20]. In normal conditions, ER-located Bcl2 interacts with BECN1 and inhibits its interaction with PIK3C3, leading to autophagy suppression [21, 22]. Upon starvation stimulation, Bcl2 is phosphorylated by JNK1 and then disassociates with BECN1 [23]. Thus, released BECN1 binds to PIK3C3 
to activate its kinase activity. AMBRA1 was reported to modulate the BECN1-PIK3C3 complex [13, 24-26]. In a normal condition, AMBRA1 links the BECN1PIK3C3 complex to the cytoskeleton by interacting with dynein light chain $1 / 2$ [13]. Upon autophagy induction, AMBRA1 is phosphorylated by ULK1 to release from the cytoskeletal docking site to induce autophagosome nucleation $[13,24]$. AMBRA1 can act as a substrate receptor for the TRAF6 ligase to mediate ULK1 K63linked ubiquitination [25], which potentiates ULK1 stability and activity. We recently demonstrated that WASH (Wiskott-Aldrich syndrome protein (WASP) and SCAR homolog) is a new interactor of BECN1 and component of the BECN1-PIK3C3 complex [27]. The AMBRA1-DDB1-CUL4A complex is an E3 ligase for K63-linked ubiquitination of BECN1 that enhances its association with PIK3C 3 and is required for starvation-induced autophagy. WASH suppresses the ubiquitination of BECN1 to inactivate PIK3C3 activity, leading to suppression of autophagy.

RNF2, also called Ring1B, was firstly identified as an interactor of Bmi1, a group II polycomb group (PcG) protein [28]. PcG proteins are present in two distinct core complexes, polycomb repressor complex I (PRC1) and polycomb repressor complex II [29]. RNF2 is contained in the PRC1 complex, acting as an ubiquitin E3 ligase to ubiquitinate histone $\mathrm{H} 2 \mathrm{~A}$ for its monoubiquitination [30, 31]. RNF2 deficiency causes early embryonic lethality [32], suggesting that RNF2 plays a pivotal role in early development. In addition to its monoubiquitination activity for $\mathrm{H} 2 \mathrm{~A}$, the PRC1 complex also has polyubiquitination activity. PRC1 polyubiquitinates DNA replication inhibitor Geminin to maintain the activity of adult hematopoietic stem cells [33]. A recent study showed that RNF2 also polyubiquitinates tumor suppressor TP53 in selective tumor types leading to tumor formation [34]. However, only a few target substrates of RNF2 has been identified up to date and its role in autophagy regulation is still unknown. Here, we show that RNF2 is an E3 ligase for K48-linked ubiquitination of AMBRA1. WASH can recruit RNF2 for AMBRA1 degradation, leading to downregulation of autophagy.

\section{Results}

\section{RNF2 interacts with AMBRA1}

We recently showed that WASH deficiency causes early embryonic lethality and extensive autophagy of mouse embryos [27]. We identified that WASH is a new interactor of BECN1 to inhibit autophagy through suppression of the ubiquitination of BECN1. The AMBRA1-DDB1-CUL4A complex acts as an E3 ligase for
K63-linked ubiquitination of BECN1 that augments PIK3C3 activity. However, how AMBRA1 is regulated in autophagy remains elusive.

RNF2, also known as Ring1B, was firstly identified as an interactor of Bmil [28]. It was defined as an ubiquitin E3 ligase [30, 31]. Interestingly, we found that RNF2 was present in the WASH-associated BECN1-PIK3C3 complex (data not shown). We further observed that RNF2 core resided on the WASH-associated autophagosomes (Supplementary information, Figure S1), suggesting that RNF2 is involved in autophagy regulation. To further explore how RNF2 regulates autophagy, we used RNF2 as a bait to screen a human spleen cDNA library using a yeast two-hybrid system. Interestingly, AMBRA1 was identified as an interactor of RNF2 (Figure 1A). We obtained seven AMBRA1-positive clones in the RNF2 screening. The interaction of AMBRA1 with RNF2 was further validated in co-transfected mouse embryonic fibroblast (MEF) cells. FLAG-tagged RNF2 could precipitate AMBRA1 in co-transfected mammalian cells and vice versa (Figure $1 \mathrm{~B}$ and $1 \mathrm{C}$ ). Moreover, recombinant GST-tagged RNF2 could pull down AMBRA1 from MEF lysates (Figure 1D), while the GST alone could not. In addition, the direct interaction of RNF2 with AMBRA1 was confirmed by GST pull-down assay after incubation of their recombinant proteins (Figure 1E).

Finally, we immunoprecipitated endogenous proteins by using antibodies against AMBRA1 and RNF2 under normal or starvation conditions. As expected, RNF2 weakly interacted with AMBRA1 under a normal condition (Figure 1F). However, the interaction between RNF2 and AMBRA1 was greatly enhanced upon autophagy induction (Figure 1F). In addition, MG132 treatment impeded the degradation of AMBRA1, which enhanced the interaction between RNF2 and AMBRA1. Taken together, RNF2 directly interacts with AMBRA1 and their interaction is enhanced upon autophagy induction.

\section{RNF2 deficiency augments autophagy}

To delineate the physiological role of RNF2 in autophagy, we generated RNF2 knockout (KO) MEFs by expressing Cre recombinase in $R N F 2^{\text {floxfflox }}$ MEFs. Intriguingly, in RNF2 KO MEFs, MAP1LC3B lipidation was enhanced compared to that in RNF2 WT MEFs after EBSS (Earle's balanced salt solution, a nutrient-deprived condition) treatment (Figure 2A), and autophagic substrate SQSTM1 was mostly hydrolyzed. In addition, a lysosomal inhibitor Bafilomycin A1 (BafA1) treatment further enhanced MAP1LC3B-II accumulation. However, BafA1 blocked the degradation of SQSTM1. Consistently, the number of MAP1LC3B dots was boosted in RNF2 KO cells (Figure 2B), whereas BafA1 treatment 
A
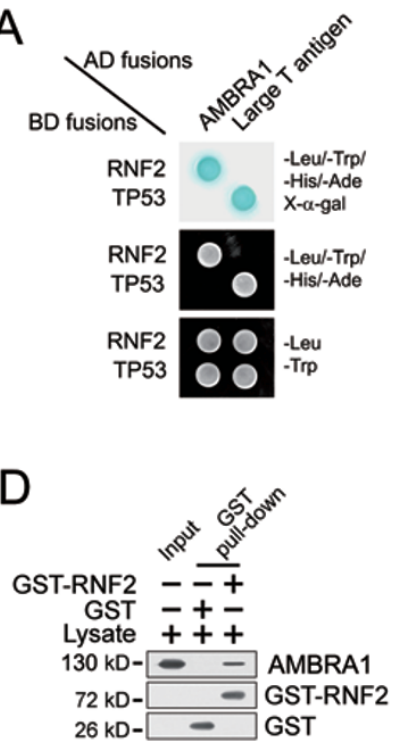

B
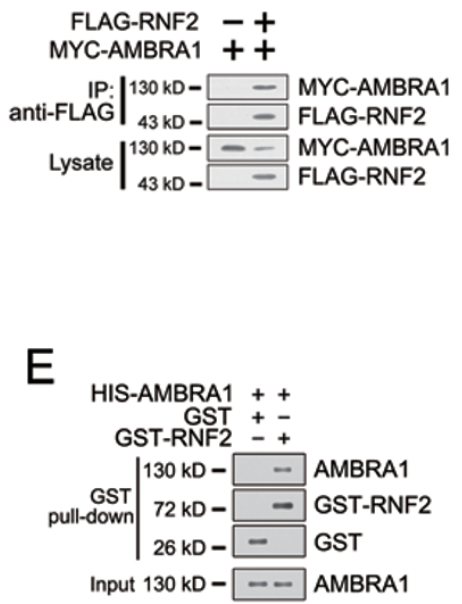

C

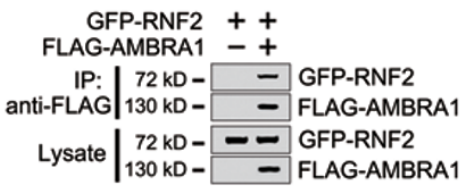

F

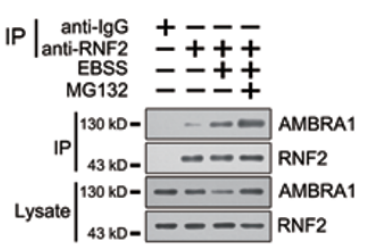

Figure 1 RNF2 interacts with AMBRA1. (A) RNF2 interacts with AMBRA1 in yeast two-hybrid assays. Yeast strain AH109 was transfected with BD (Gal4 DNA-binding domain)-fused RNF2 and AD (Gal4 activating domain)-fused AMBRA1 and plated on the indicated medium for $3 \mathrm{~d}$. TP53 with large T antigen was introduced as a positive control. (B, C) RNF2 interacts with AMBRA1 in mammalian cells. FLAG-tagged RNF2 and MYC-tagged AMBRA1 were co-transfected into MEF cells for 24 h, followed by IP with antibody against FLAG (B). GFP-tagged RNF2 and FLAG-tagged AMBRA1 were co-transfected into MEF cells for $24 \mathrm{~h}$, followed by IP with antibody against FLAG (C). (D) GST-RNF2 precipitates AMBRA1 from MEF cell lysates. GST-RNF2 or GST was bound to GST beads and incubated with MEF cell lysates, followed by immunoblotting with the indicated antibodies. (E) RNF2 directly interacts with AMBRA1. Recombinant GST-tagged RNF2 were incubated with HIS-AMBRA1, followed by GST pull-down assays. (F) Endogenous RNF2 interacts with AMBRA1 upon starvation. MEFs were treated with culture medium (CM) or EBSS for $2 \mathrm{~h}$, followed by IP with antibody against RNF2. $10 \mu \mathrm{M}$ MG132 was added to cell culture before EBSS treatment. Experiments were repeated three times with similar results.

remarkably increased MAP1LC3B puncta. These data suggest that RNF2 deficiency causes a robust autophagic activity with autophagic influx. Furthermore, the number of GFP-ATG5 dots was increased in RNF2-deficient cells compared to RNF2 WT cells with EBSS treatment (Figure 2C). MAP1LC3B puncta and GFP-ATG5 dots represent autophagosome formation.

We next examined the degradation of long-lived proteins in $R N F 2^{+/+}$and $R N F 2^{-/-}$cells. We observed that much more long-lived proteins were degraded in $R N F 2^{-/-}$cells than in $R N F 2^{+/+}$cells after EBSS treatment (Figure 2D). A type III PI3K inhibitor 3-MA, blocking autophagosome formation, impaired the degradation of long-lived proteins (Figure 2D). Importantly, upon EBSS treatment, $R N F 2^{-}$MEFs exhibited substantial autophagosome-like structures by electronic microscopy (Figure 2E). Consistently, MAP1LC3B conversion was increased and SQSTM1 degradation was accelerated in RNF2 KO MEFs compared to RNF2 WT MEFs (data not shown). These results suggest that RNF2 deficiency enhances autophagy induction.

Histidine 69 to tyrosine mutation (H69Y) of RNF2 abolishes its ligase activity, which is called the ligase-dead RNF2 (H69Y-RNF2) [35]. We then overexpressed RNF2 or H69Y-RNF2 mutant in MEFs to verify its inhibitory role in autophagy. We found that the exogenous expression levels of RNF2 were comparable to that of WT MEFs (Figure 2F, left panel). When starved with EBSS for $4 \mathrm{~h}$, RNF2-restored MEFs showed reduced conversion of MAP1LC3B-II compared with vector-transfected MEFs (Figure 2F). In RNF2-restored cells, the degradation of SQSTM1 was almost blocked (Figure 2F, right panel). However, the overexpression of ligase-dead mutant H69Y-RNF2 did not affect autophagy. Consistently, RNF2 overexpression dramatically suppressed the formation of MAP1LC3B dots compared to vector transfection (Figure 2G). These data indicate that RNF2 inhibits autophagy and this suppressive role requires its enzymatic activity.

RNF2 promotes AMBRA1 degradation upon autophagy induction

AMBRA1 was identified as a positive regulator of the BECN1-PIK3C3 complex to induce autophagy [13]. We 
A
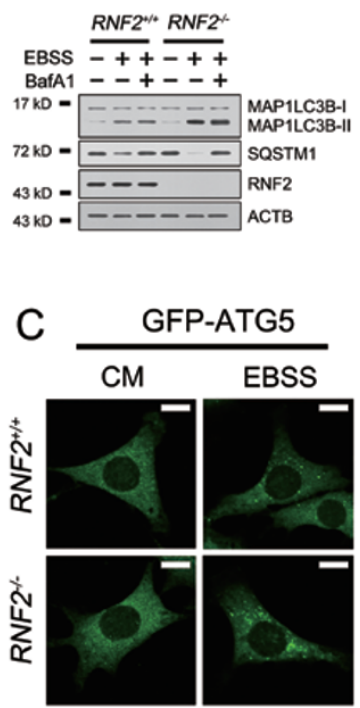

$\mathrm{F}$
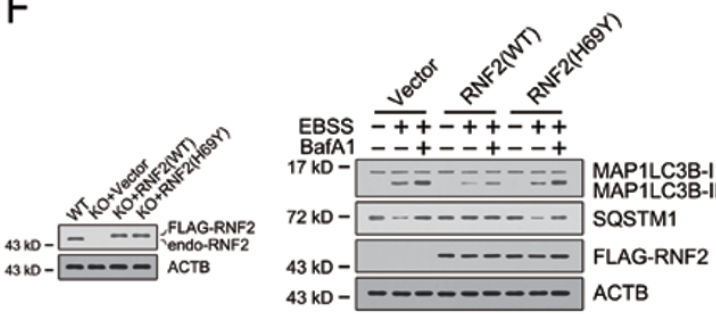

B

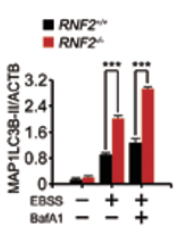

MAP1LC3B

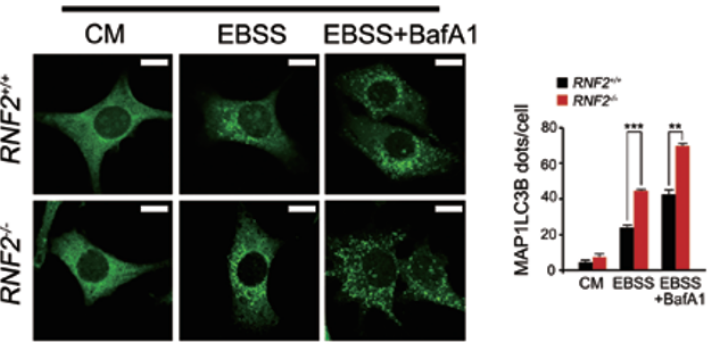

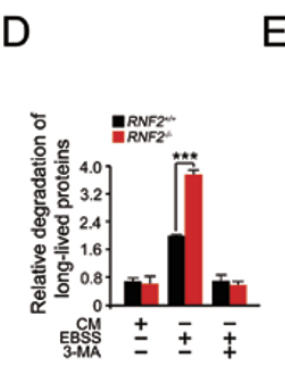

E

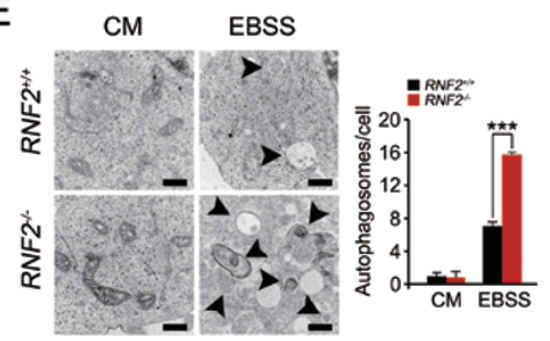

G

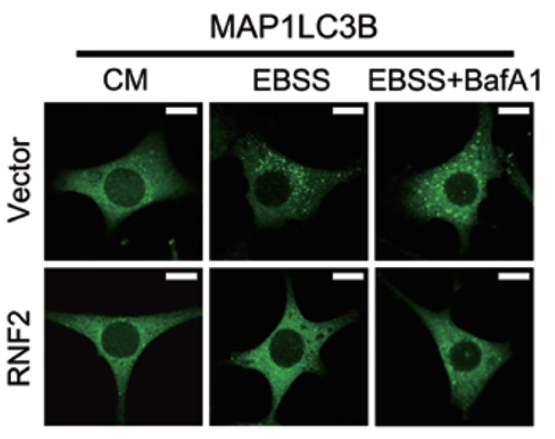

Figure 2 RNF2 suppresses autophagy. (A) RNF2 deficiency leads to enhanced autophagic activity. WT control $\left(R N F 2^{+/+}\right)$and RNF2 KO (RNF2 $\left.{ }^{--}\right)$MEFs were starved in EBSS for $4 \mathrm{~h}$ with or without BafA1. Lysates were analyzed by immunoblotting with the indicated antibodies (left panel) and the ratio of MAP1LC3B-II/ACTB was calculated (right panel). (B) MAP1LC3B dots were increased in RNF2 KO cells. RNF2 ${ }^{+/+}$and $R N F 2^{-/-}$ MEF cells were starved in EBSS for $4 \mathrm{~h}$ with or without BafA1. Endogenous MAP1LC3B was stained with antibody against MAP1LC3B and visualized by confocal microscopy (left panel). The number of MAP1LC3B dots/puncta was counted from at least 100 cells for each condition (right panel). Scale bar, $10 \mu \mathrm{m}$. (C) ATG5 dots are increased in RNF2 $2^{-/-}$MEFs. RNF2 $2^{+/+}$and $\mathrm{RNF2}^{-/-}$ MEFs stably expressing GFP-ATG5 were stimulated with EBSS for 4 h. GFP-ATG5 dots were visualized and counted as B. (D) RNF2 deficiency increases degradation of long-lived proteins in MEFs under starvation conditions. 3-MA, a type III phosphatidylinositol 3-kinases (PI-3K) inhibitor, was added to block the activity of autophagy. (E) RNF2-deficient MEFs exhibit enhanced autophagy. $\mathrm{RNF2}^{+/+}$and $R N F 2^{-/-} \mathrm{MEFs}$ were treated with culture medium (CM) or EBSS for $4 \mathrm{~h}$, followed by examination with transmission electron microscopy. Black arrowhead indicates

previously showed that the DDB1-CUL4A-AMBRA1 complex acts as an ubiquitin E3 ligase for K63-linked ubiquitination of BECN1, which enhances the PIK3C3 kinase activity [27]. As shown above, RNF2 is a new interactor of AMBRA1 and inhibits autophagy. We next wanted to test how RNF2 regulates AMBRA1 in autoph- 
agy. We found that AMBRA1 was degraded in $R N F 2^{+/+}$ cells during EBSS treatment (Figure 3A). However, the degradation of AMBRA1 was totally blocked in $\mathrm{RNF}^{-1}$ cells. Moreover, starvation-induced autophagy activity was augmented in $R N F 2^{-/-}$cells (Figure $3 \mathrm{~A}$ ). In addition, the half-life of AMBRA1 in $R N F 2^{+/+}$cells was around $4 \mathrm{~h}$ after EBSS treatment, whereas AMBRA1 remained stable in $R_{N F 2^{-/}}$cells even by $8 \mathrm{~h}$ (Figure 3B). However, RNF2 deficiency did not affect the mRNA levels of AMBRA1, as well as other autophagy-related genes that we examined, such as PIK3C3 and BECN1 (Figure $3 \mathrm{C})$. These data suggest that RNF2 accelerates AMBRA1 degradation during starvation-induced autophagy.

To exclude the possibility that the AMBRA1 degradation is caused by an indirect effect of RNF2 KO, we performed rescue experiments by introducing RNF2 or H69Y-RNF2 to $R N F 2^{-/-}$MEFs. RNF2 restoration in $R N F 2^{-/-}$cells resulted in rapid degradation of AMBRA1 after EBSS treatment (Figure 3D), while empty vector-introduced $R N F 2^{-/-}$cells sustained the AMBRA1 stability. Importantly, H69Y-RNF2 restoration had no such effect (Figure 3D). These data indicate that RNF2 directly accelerates the degradation of AMBRA1.

Proteins are degraded mainly through a proteasome
A

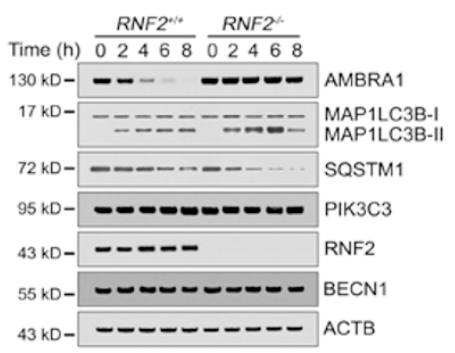

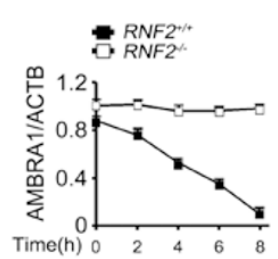
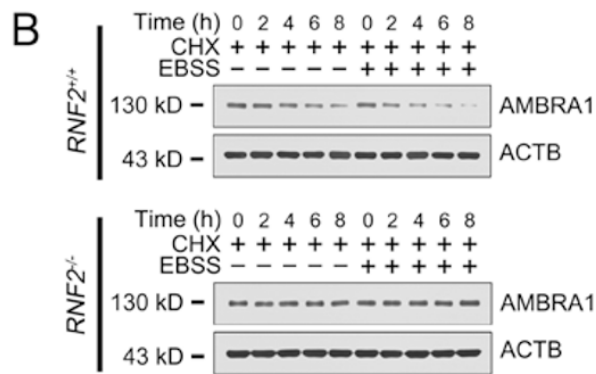
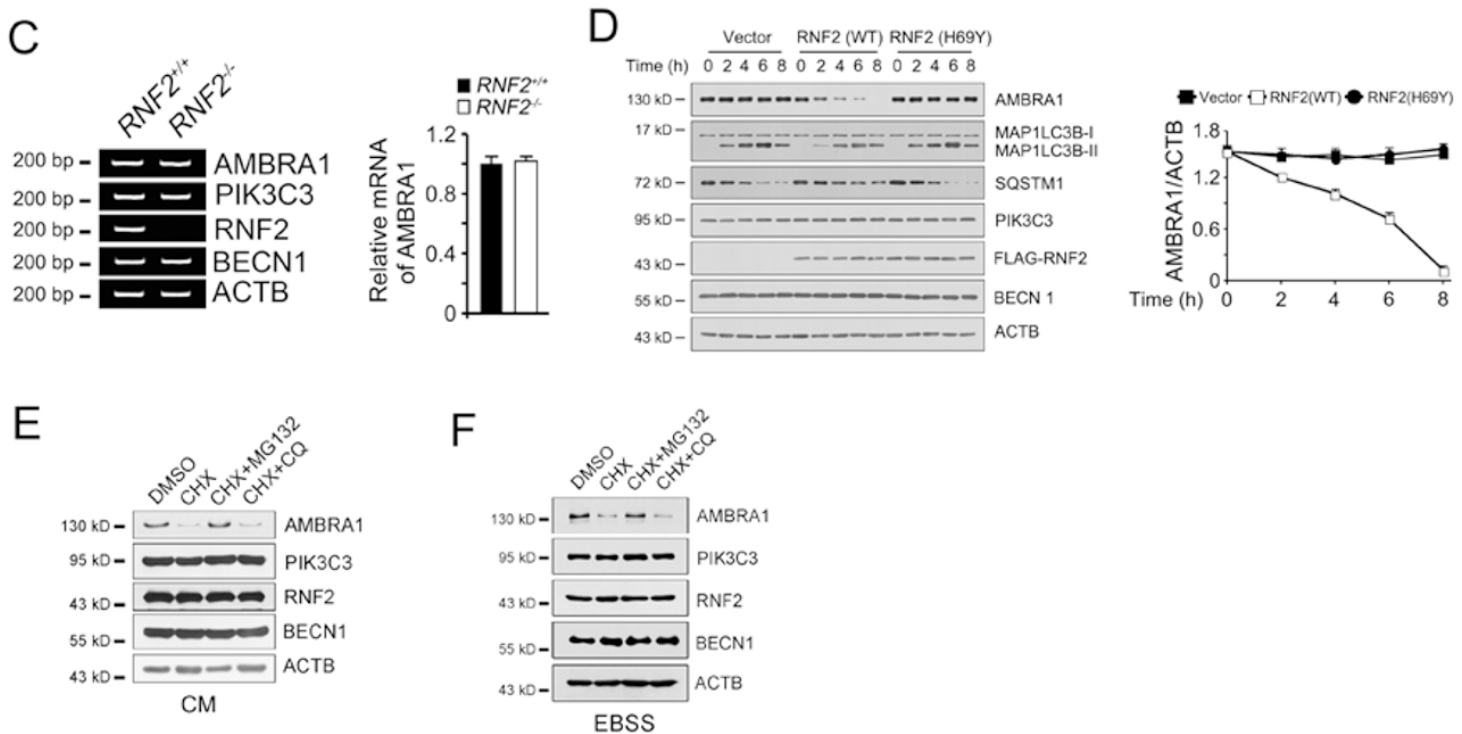

Figure 3 RNF2 promotes AMBRA1 degradation through a proteasome degradation pathway during autophagy. (A) RNF2 deficiency prevents degradation of AMBRA1. RNF2 ${ }^{+/}$and $R N F 2^{-/-} \mathrm{MEFs}$ treated with EBSS for the indicated times were harvested for immunoblotting (left panel). Ratios of AMBRA1/ACTB were calculated (right panel). (B) The half-life of AMBRA1.

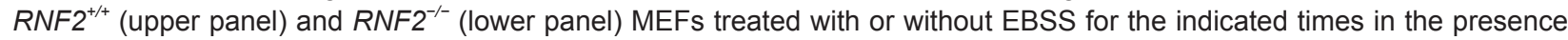
of $20 \mu \mathrm{g} / \mathrm{ml}$ cycloheximide (CHX) were harvested for immunoblotting with the indicated antibodies. (C) RNF2 deficiency does not change the mRNA level of AMBRA1. RNF2 ${ }^{+/+}$and $R N F 2^{-/-}$MEFs were harvested, and mRNA levels for the indicated genes were analyzed by RT-PCR. mRNA levels of AMBRA1 were normalized to those of ACTB (right panel). (D) RNF2 restoration in $\mathrm{RNF2}^{-/-} \mathrm{MEFs}$ rescues the degradation of AMBRA1 during autophagy. $\mathrm{RNF}^{-/-} \mathrm{MEFs}$ were infected with lentiviruses encoding WT- or H69Y-RNF2 and treated with EBSS, followed by immunoblotting (left panel). Ratios of AMBRA1/ACTB were calculated (right panel). (E, F) AMBRA1 is degraded through a proteasome degradation pathway. MEFs were treated with the

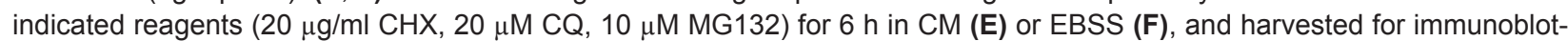
ting. Data are representative of at least three separate experiments and shown as means \pm SD. 
pathway or a lysosome pathway in eukaryotic cells [3, 36]. The proteasomal pathway recognizes only ubiquitinated substrates for degradation. In contrast, extracellular materials and cytosolic components can be delivered to the lysosome for degradation. MG132 is an inhibitor of the proteasome pathway, whereas chloroquine (CQ) is an inhibitor of the lysosome pathway. MG132 treatment blocked the degradation of AMBRA1 in a normal condition, while CQ had no such blocking activity (Figure $3 \mathrm{E})$. To exclude the possibility that AMBRA1 was a target substrate of autophagy for its degradation, we performed the above experiments in starvation conditions. AMBRA1 was hydrolyzed with EBSS treatment (Figure 3F). Moreover, MG132 could still block the degradation of AMBRA1, while CQ had no such effect (Figure 3F). These data indicate that AMBRA1 is degraded through the proteasome pathway, but not the lysosome pathway.

We separated the nuclei and cytoplasm of MEFs to examine the distribution of RNF2. We observed that RNF2 localized in both the nucleus and the cytoplasm (Supplementary information, Figure S2A). Upon starvation, the cytosolic AMBRA1 underwent degradation, whereas the nuclear AMBRA1 remained stable after EBSS treatment. These data demonstrated that degradation of AMBRA1 occurs in the cytoplasm after starvation.

\section{RNF2 is an E3 ligase for K48-linked ubiquitination of AMBRA1}

RNF2 was identified as a member of the RING-domain E3 family that is present in the PRC1 complex [32]. RNF2 can ubiquitinate histone H2A for its monoubiquitination [30, 31]. RNF2 also ubiquitinates Geminin and TP53 for polyubiquitination $[33,34]$. To test whether RNF2 is an E3 ligase for AMBRA1 ubiquitination, we first co-transfected AMBRA1 with increasing amounts of RNF2 into MEF cells. RNF2 overexpression resulted in declined protein levels of AMBRA1 (Figure 4A), and this degradation was RNF2 dose-dependent. However, RNF2 overexpression did not influence the mRNA level of AMBRA1 (Figure 4B). These data indicate that RNF2 promotes AMBRA1 degradation at the protein level. Thus, we proposed that RNF2 might be an E3 ligase for AMBRA1 degradation.

We then transfected empty vector, WT RNF2 or H69YRNF2 mutant with ubiquitin mutants into RNF2-deficient MEFs. We found that WT RNF2 overexpression with K63R-Ub, but not K48R-Ub, led to polyubiquitination of AMBRA1 (Figure 4C). However, overexpression of the ligase-dead H69Y-RNF2 with K63R-Ub or K48R-Ub failed to mediate polyubiquitination of AMBRA1 (Figure 4C). Consistently, AMBRA1 ubiquitination caused its degradation, which led to the suppression of autophagy
(Figure 4C). In contrast, H69Y-RNF2 did not cause AMBRA1 ubiquitination, which sustained the stability of AMBRA1 and autophagy similar to empty vector-transfected control cells. In addition, overexprssion of RNF2 or H69Y-RNF2 did not affect the mRNA level of AMBRA1. These results suggest that RNF2 catalyzes K48linked ubiquitination of AMBRA1.

To directly confirm that RNF2 is an E3 ligase of AMBRA1, we generated recombinant proteins of RNF2, AMBRA1, and components of the ubiquitin-proteasome system for an in vitro ubiquitination reconstitution assay. Expectedly, AMBRA1 was polyubiquitinated in the presence of RNF2 and K63R-Ub in the reconstitution system (Figure 4D), and this ubiquitination was abolished in the presence of K48R-Ub. Finally, we examined the ubiquitination levels of AMBRA1 in $R N F 2^{+/+}$and $R N F 2^{-/-}$cells with overexpression of ubiquitin mutants. We observed that the ubiquitination of AMBRA1 was blocked in the presence of K48R-Ub in $R N F 2^{+/+}$MEFs upon EBSS treatment (Figure 4E), whereas polyubiquitination of AMBRA1 was detected in the presence of K63R-Ub. However, the K48-linked ubiquitination of AMBRA1 was totally abolished in RNF2 KO MEFs. In sum, RNF2 is an E3 ligase for AMBRA1 to catalyze K48-linked polyubiquitination for its degradation during autophagy.

\section{RNF2 ubiquitinates AMBRA1 at lysine 45}

We next determined the ubiquitination sites on AMBRA1. We generated several truncations of AMBRA1 (Figure 5A) and transfected them with RNF2 into MEF cells. After starvation in EBSS for $2 \mathrm{~h}$, cell lysates were immunoprecipitated with anti-AMBRA1 antibody and the ubiquitination state of these truncations was examined. Interestingly, the first 50 amino acid truncation $(\Delta 1$ 50) was not ubiquitinated (Figure 5B), while the other two truncations $(\Delta 60-180$ and $\Delta 760-980)$ were ubiquitinated. We predicted that the ubiquitination sites on AMBRA1 were localized at the first 50 amino acids of the $\mathrm{N}$ terminus. We further mutated all the lysines to arginines within the first 50 amino acids of AMBRA1. We found that K45R-AMBRA1 mutation totally abolished the ubiquitination of AMBRA1 in the presence of RNF2 (Figure 5C), whereas other mutants (K2R, K7R, K36R and $\mathrm{K} 41 \mathrm{R}$ ) were ubiquitinated. These data indicate that K45 on AMBRA1 is the ubiquitination site for RNF2.

\section{K45R-AMBRA1 mutant sustains its stability to enhance PIK3C3 activity}

We then established AMBRA1-silenced MEFs and rescued WT-AMBRA1 or K45R-AMBRA1 expression in these AMBRA1-depleted cells. We found that the K45R-AMBRA1 overexpression completely blocked 
A
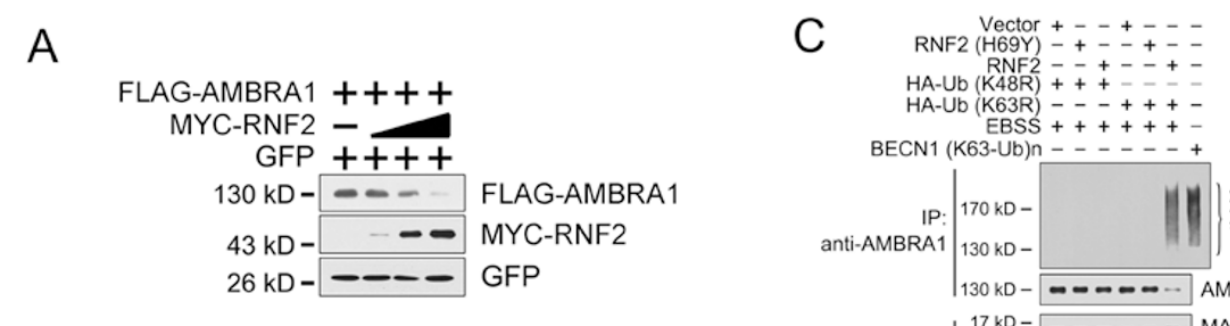

B
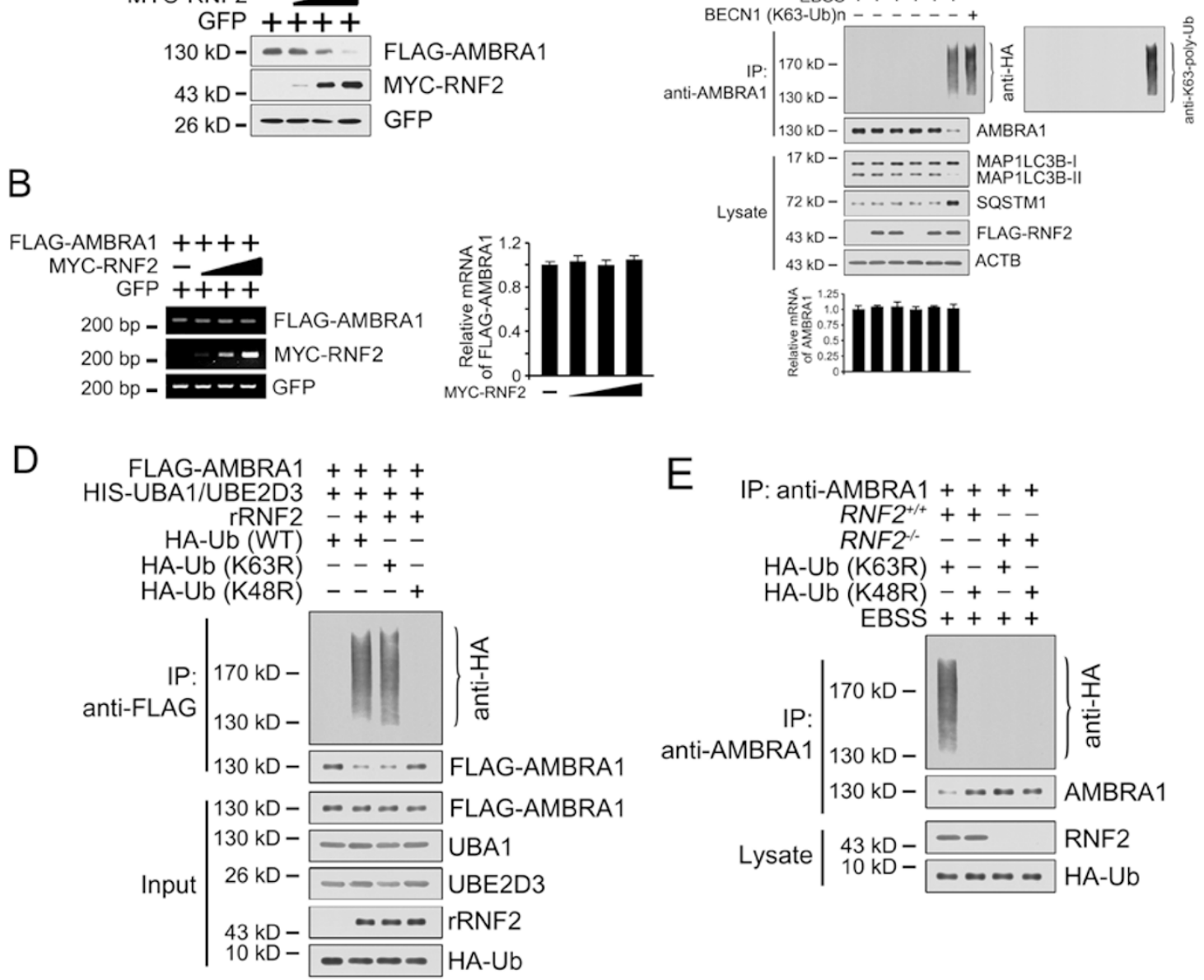

Figure 4 RNF2 is an E3 ligase for K48-linked ubiquitination of AMBRA1. (A, B) RNF2 promotes degradation of AMBRA1. FLAG-tagged AMBRA1 and increasing amounts of MYC-tagged RNF2 were co-transfected into MEF cells for $24 \mathrm{~h}$. The protein levels were examined by immunoblotting with the indicated antibodies (A). The mRNA levels were examined by RT-PCR with primers specific for exogenous plasmids (B, left panel). mRNA levels of AMBRA1 were normalized to those of GFP (B, right panel). (C) RNF2 overexpression enhances K48-linked ubiquitination of AMBRA1. RNF2 ${ }^{-/}$MEFs transfected with the indicated RNF2 plasmids and ubiquitin mutants were starved in EBSS for $2 \mathrm{~h}$ in the presence of $10 \mu \mathrm{M}$ MG132, followed by IP with antibody against AMBRA1. The immunoprecipitates were dissociated with $1 \%$ SDS and reimmunoprecipitated with anti-AMBRA1. Immunoprecipitates and lysates were immunoblotted with the indicated antibodies. K63-polyubiquitinated BECN1 was immunoprecipitated with antibody against BECN1 from starved cells stably expressing HA-Ub as a positive control for antibody against K63-linked polyubiquitin chains. The mRNA level of AMBRA1 was examined by RT-PCR. (D) RNF2 ubiquitinates AMBRA1 via K48 linkage by in vitro ubiquitination reconstitution assays. Recombinant RNF2 was incubated with purified FLAG-AMBRA1 in the presence of recombinant E1 (UBA1) and E2 (UBE2D3) and ubiquitin mutants at $30^{\circ} \mathrm{C}$ for $1 \mathrm{~h}$, followed by IP with antibody against FLAG and immunoblotting with the indicated antibodies. (E) RNF2 deficiency blocks the ubiquitination of AMBRA1 during autophagy. $R N F 2^{+/+}$and $R N F 2^{-/-} \mathrm{MEFs}$ transfected with various ubiquitin mutants were starved in EBSS for $2 \mathrm{~h}$ in the presence of $10 \mu \mathrm{M}$ MG132, followed by IP with antibody against AMBRA1. Data represent at least three separate experiments.

its ubiquitination under starvation (Figure 5D), whereas WT-AMBRA1 was able to undergo ubiquitination. Consistently, K45R-AMBRA1 overexpression maintained the stability of AMBRA1 and enhanced autophagy (Fig- ure 5D), while WT-AMBRA1 restoration resulted in its degradation and moderate autophagy. Moreover, the halflife of AMBRA1 was around $4 \mathrm{~h}$ in WT-AMBRA1-restored cells upon EBSS treatment (Figure 5E), which 
A

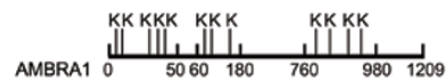

D

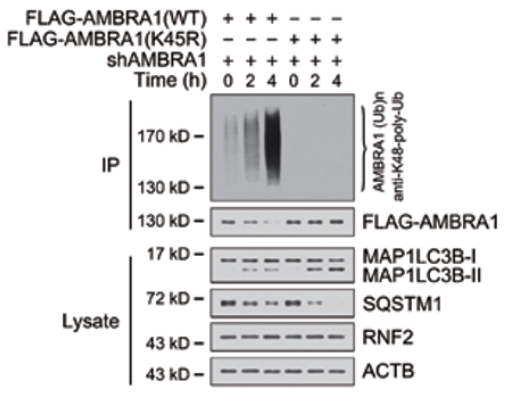

B

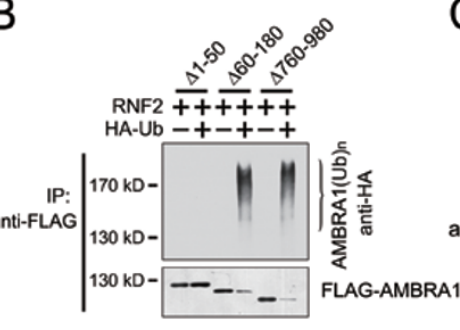

E
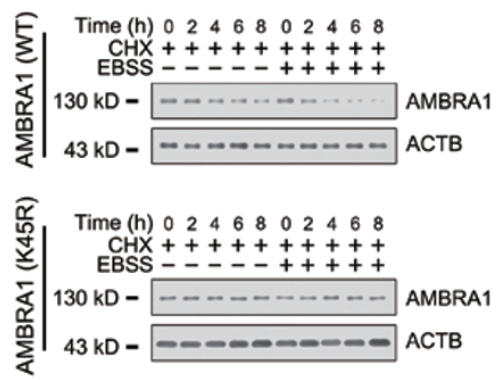

G

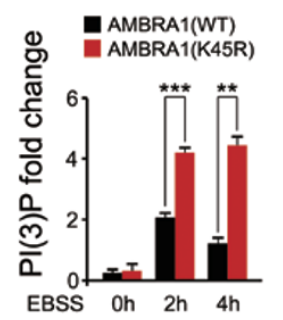

I

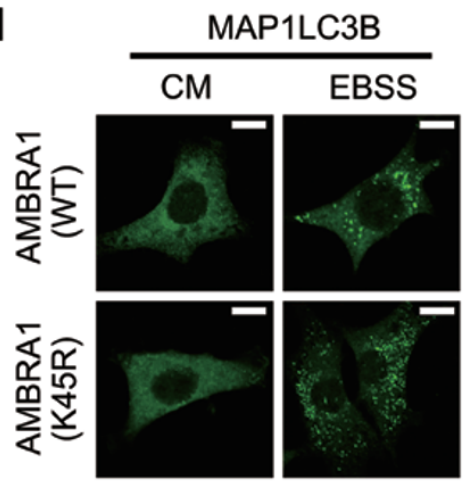

$\mathrm{H}$

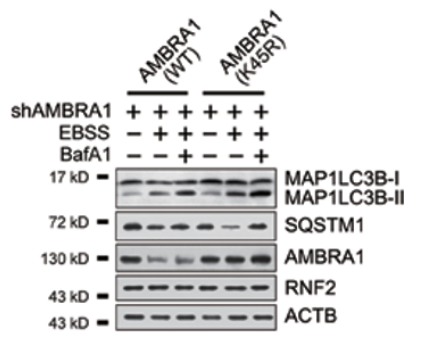

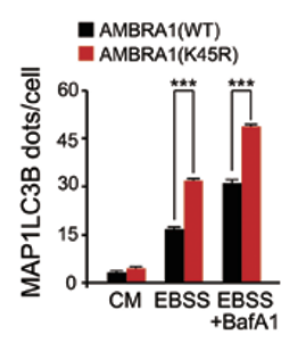

J
C

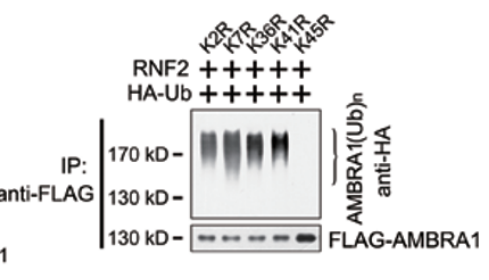

F
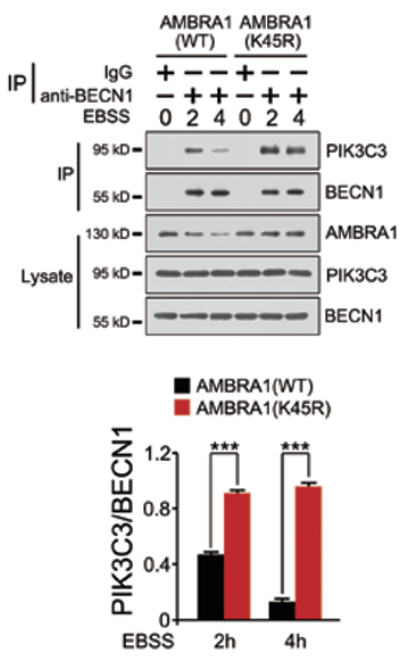

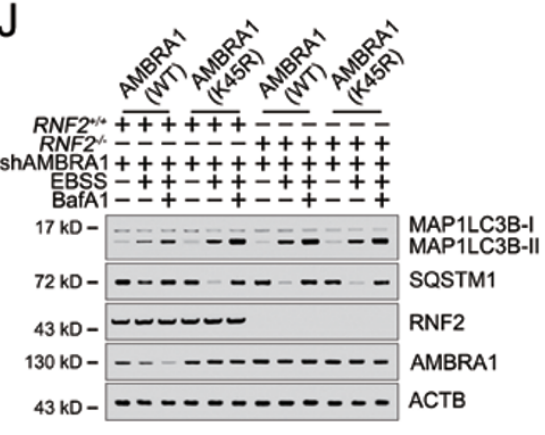

Figure 5 K45R-AMBRA1 mutant maintains AMBRA1 stability and promotes PIK3C3 activity. (A) Scheme for lysines on AMBRA1. (B, C) K45 is the RNF2-mediated ubiquitination site on AMBRA1. Various AMBRA1 truncations (B) or mutants (C) were co-transfected with RNF2 with or without HA-tagged ubiquitin (HA-Ub) into MEFs for $24 \mathrm{~h}$. Cells were harvested after pretreatment with $10 \mu \mathrm{M}$ MG132 for $6 \mathrm{~h}$ and EBSS for $2 \mathrm{~h}$, followed by IP with antibody against AMBRA1. The immunoprecipitates were dissociated with $1 \%$ SDS and reimmunoprecipitated with anti-FLAG. Immunoprecipitates were immunoblotted with the indicated antibodies. (D) K45R-AMBRA1 enhances autophagy. AMBRA1-silenced MEFs were stably rescued with WT-AMBRA1 or K45R-AMBRA1 and starved in EBSS for the indicated times, followed by IP with antibody against FLAG after pretreated with $10 \mu \mathrm{M}$ MG132 for $6 \mathrm{~h}$. Immunoprecipitates were immunoblotted with the indicated antibodies. (E) The halflife of AMBRA1. AMBRA1-silenced MEFs were transfected with WT-AMBRA1 (upper panel) or K45R-AMBRA1 (lower panel) and selected for stable expression with $2 \mu \mathrm{g} / \mathrm{ml}$ puromycin. Cells were then treated with or without EBSS for the indicated times in the presence of $20 \mu \mathrm{g} / \mathrm{ml} \mathrm{CHX}$ before harvest for immunoblotting with the indicated antibodies. (F) The association of BECN1 with PIK3C3 is enhanced in K45R-AMBRA1-rescued cells. AMBRA1-silenced MEFs were rescued with WT-AMBRA1 or K45R-AMBRA1 for $24 \mathrm{~h}$, followed by IP with anti-BECN1 antibody. Immunoprecipitates were immunoblotted with the indicated antibodies (upper panel). The ratios of PIK3C3 versus BECN1 were calculated and shown in the lower panel. (G) 
was consistent with EBSS-treated WT RNF2 cells (Figure 3B). However, K45R-AMBRA1 restoration did not cause degradation of AMBRA1 with EBSS treatment. Furthermore, the amount of $\mathrm{BECN} 1$-associated $\mathrm{PIK} 3 \mathrm{C} 3$ protein was dramatically increased in K45R-AMBRA1-rescued cells (Figure 5F). Consequently, the BECN1 associated-PIK3C3 kinase activity was also enhanced in K45R-AMBRA1-rescued cells (Figure 5G).

We then examined the autophagy activity in WT-AMBRA1- and K45R-AMBRA1-rescued cells. We found that K45R-AMBRA1 overexpression accelerated MAP1LC3B lipidation and SQSTM1 degradation (Figure $5 \mathrm{H})$, and BafA1 treatment further enhanced MAP1LC3B accumulation but blocked SQSTM1 degradation. In contrast, WT-AMBRA1-transfected cells only had moderate MAP1LC3B conversion and SQSTM1 degradation (Figure $5 \mathrm{H})$. Moreover, the number of MAP1LC3B puncta was remarkably increased in K45R-AMBRA1-rescued cells compared to that of WT-AMBRA1-transfected cells (Figure 5I). Finally, K45R-AMBRA1 restoration in RNF2-deficient MEFs led to similar autophagy level to K45R-AMBRA1 restoration in WT MEFs (Figure 5J). These data suggest that RNF2 negatively regulates autophagy induction through ubiquitination of AMBRA1.

The Atg14-associated BECN1-PIK3C3 complex is autophagy related $[15,16]$. We performed immunoprecipitation (IP) assays by using antibodies against ATG14 and UVRAG in WT AMBRA1- or mutant AMBRA1-rescued MEF cells (Supplementary information, Figure S2B). We found that AMBRA1 mutation did not affect the interaction between BECN1 and PIK3C3 within the UVRAG-containing PIK3C3 complex. However, AMBRA1 mutation enhanced the interaction between BECN1 and PIK3C3 within the Atg14-containing PIK3C3 complex. Moreover, RNF2 deficiency enhanced the association between BECN1 and PIK3C3 within the ATG14-containing PIK3C3 complex, but not in the UVRAG-containing PIK3C3 complex. Thus, RNF2-mediated autophagy regulation involves the Atg14-associated BECN1-PIK3C3 complex.
RNF2 inhibits autophagy through promoting AMBRA1 degradation

AMBRA1 was reported to enhance the interaction between BECN1 and PIK3C3 during autophagy [13]. We showed that the DDB1-CUL4A-AMBRA1 complex acts as an E3 ligase to ubiquitinate BECN1 to augment the PIK3C3 kinase activity [27]. Thus, AMBRA1 functions as an autophagy inducer. To test whether RNF2 exerts its inhibitory role in autophagy by suppressing the association of BECN1 with PIK3C3 through modulation of AMBRA1, we examined the amount of PIK3C3 protein that was associated with BECN1 in RNF2-overexpressing cells. As expected, the amount of BECN1-associated PIK3C3 was significantly decreased in RNF2-overexpressing cells after starvation (Figure 6A). Consistently, the BECN1-associated PIK3C3 kinase activity was also declined to a lower level in RNF2-overexpressing cells compared with vector-transfected control cells (Figure $6 \mathrm{~B})$. In contrast, the amount of BECN1-associated PIK3C3 protein was higher in $R N F 2^{-/-}$cells than in $R N F 2^{+/+}$cells (Figure 6C). In addition, the kinase activity of the autophagic PIK3C3 was also higher in $R N F 2^{--}$ cells compared with $R N F 2^{+/+}$WT cells (Figure 6D).

More importantly, the amount of AMBRA1 that was associated with BECN1 was dramatically increased in RNF2 KO cells (Figure 6E). Furthermore, RNF2 deficiency significantly increased K63-linked ubiquitination of BECN1 upon autophagy induction (Figure 6F and Supplementary information, Figure S3A). In contrast, RNF2 restoration in RNF2 KO cells dramatically reduced the K63-linked ubiquitination of BECN1 (Supplementary information, Figure S3B). In addition, K437RBECN1 mutation was resistant to RNF2 deletion-induced enhancement of autophagy (Supplementary information, Figure S3C). These data suggest that RNF2-mediated AMBRA1 degradation represses K63-polyubiquitination of BECN1, leading to autophagy suppression.

WIPI1 is a PI3P-binding protein that is involved in phagophore formation [37]. We then examined WIPI1 expression levels to analyze the kinase activity of PIK3C3

K45R-AMBRA1 augments PIK3C3 kinase activity. AMBRA1-silenced MEFs were rescued with WT-AMBRA1 or K45R-AMBRA1, followed by starvation in EBSS for the indicated times. Cells were lysed for immunoblotting with anti-ATG14 antibody for autophagy-specific PIK3C3. Immunoprecipitates were split into two equal parts, one for a loading control and the other for in vitro kinase assay. Radioactive PI(3)P was resolved by thin layer chromatography (TLC), and normalized against the BECN1-associated PIK3C3. (H, I) K45R-AMBRA1 promotes autophagic activity. AMBRA1-silenced MEFs were rescued with WT-AMBRA1 or K45R-AMBRA1, followed by starvation in EBSS for $2 \mathrm{~h}$ with or without $20 \mathrm{nM}$ BafA1. Cells were lysed for immunoblotting with the indicated antibodies $(\mathbf{H})$. Endogenous MAP1LC3B were stained with antibody against MAP1LC3B and MAP1LC3B dots were counted (I). (J) RNF2 deficiency does not affect the K45R-AMBRA1-elevated autophagy. AMBRA1-silenced MEFs were stably rescued with WT-AMBRA1 or K45R-AMBRA1, followed by starvation in EBSS for $2 \mathrm{~h}$ with or without $20 \mathrm{nM}$ BafA1. Cells were lysed for immunoblotting with the indicated antibodies. Data are representative of at least three separate experiments and shown as means \pm SD. Scale bar, $10 \mu \mathrm{m}$. ${ }^{* *} P<0.01$; ${ }^{* *} P<0.001$. 
A

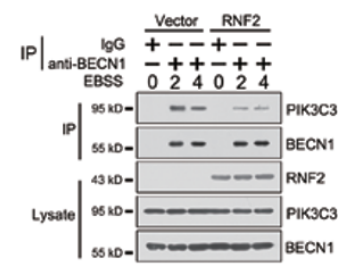

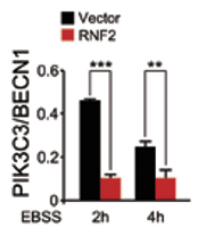

B

D

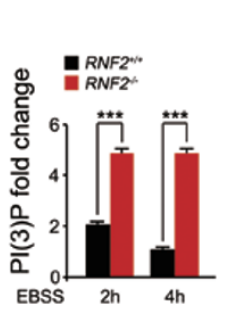

E
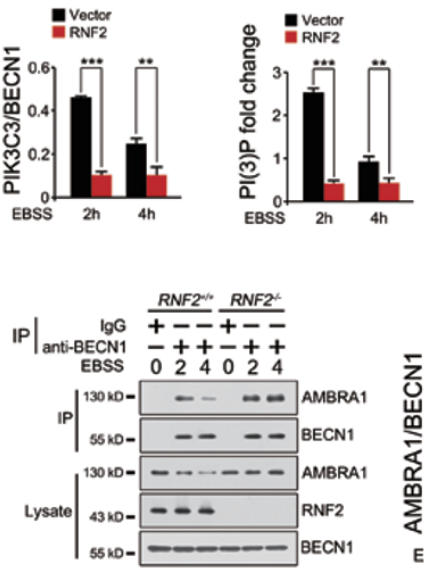

C
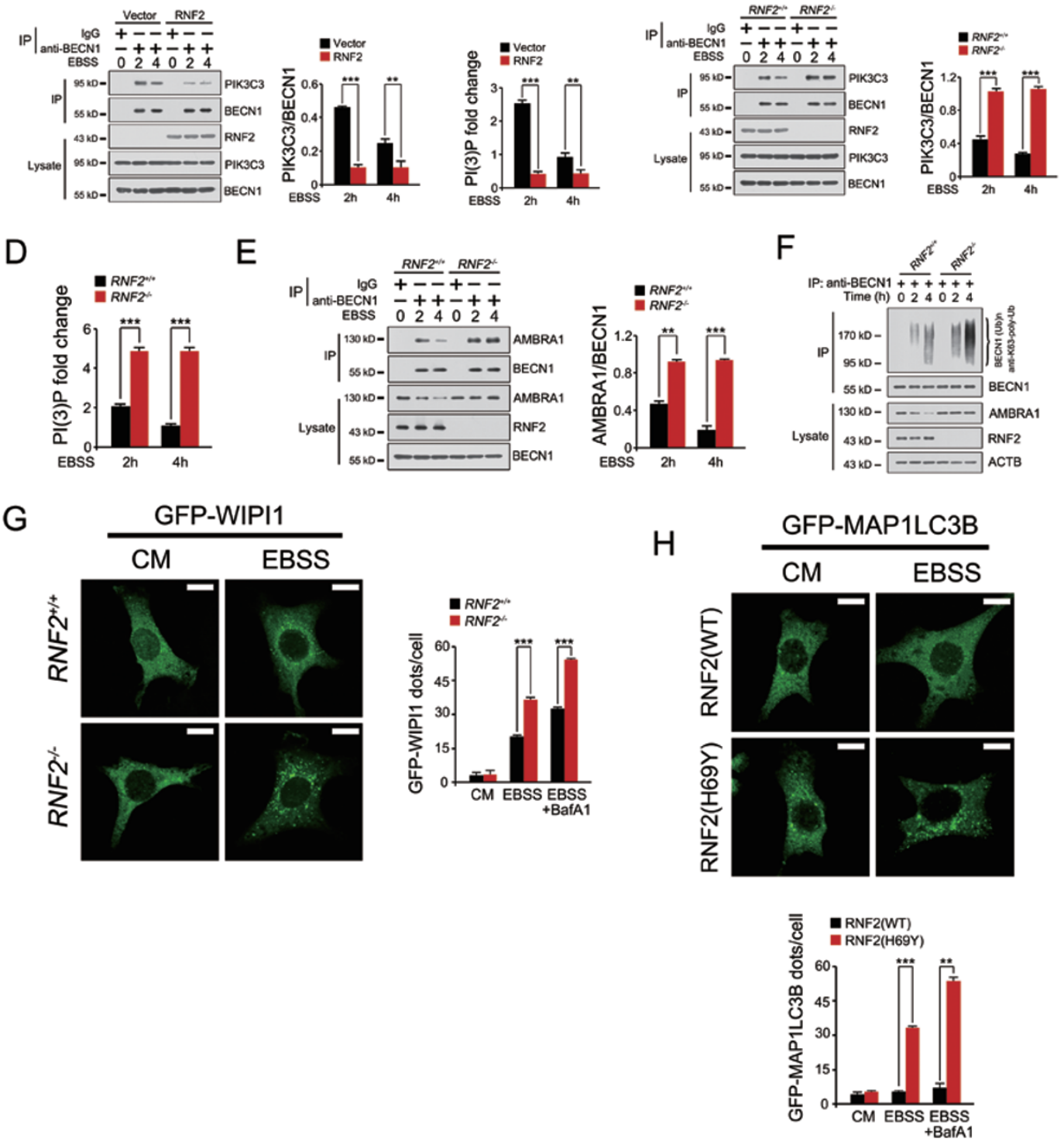

Figure 6 RNF2 inhibits autophagy through promoting AMBRA1 degradation. (A) RNF2 suppresses the association between BECN1 and PIK3C3. MEFs transfected with vector or RNF2 were starved in EBSS for the indicated times, followed by IP with anti-BECN1 antibody. Ratios of PIK3C3 versus BECN1 were calculated and shown in the right panel. (B) RNF2 inhibits the PIK3C3 kinase activity. RNF2-overexpressing MEFs were starved in EBSS for the indicated times, followed by IP with anti-ATG14 antibody for autophagy-specific PIK3C3. Immunoprecipitates were split into two equal parts and analyzed as Figure 5G. (C) RNF2 deficiency enhances the association between BECN1 and PIK3C3. RNF2 ${ }^{+/+}$and $R N F 2^{-/-}$MEFs were starved in EBSS for the indicated times, followed by IP with anti-BECN1 antibody and assayed as A. (D) RNF2 deficiency promotes the PIK3C3 kinase activity. RNF2 ${ }^{+/+}$and $R N F 2^{-/-}$MEFs were starved in EBSS for the indicated times, and harvested for IP with anti-ATG14 antibody for autophagy-specific PIK3C3. Immunoprecipitates were analyzed as B. (E) RNF2 deficiency increases the amount of AMBRA1 that is associated with BECN1. RNF2 ${ }^{+/+}$and $R N F 2^{-/-}$MEFs were incubated with EBSS for the indicated times and harvested for IP with anti-BECN1 antibody (left panel). Ratios of AMBRA1/BECN1 were analyzed and shown in the right panel. (F) RNF2 deficiency increases K63-linked ubiquitination of BECN1 during autophagy. RNF2 ${ }^{+/+}$and $R N F 2^{-/-}$MEFs were starved in EBSS for the indicated times, and harvested for IP with antibody against BECN1. Immunoprecipitates and cell lysates were immunoblotted with the indicated antibodies. (G) RNF2 deficiency elevates the kinase activity of PIK3C3. RNF2/+ and

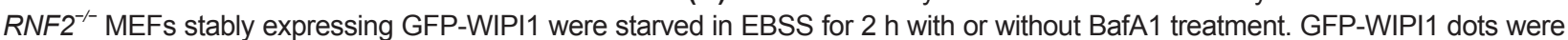
counted and shown in the right panel. (H) H69Y-RNF2 mutation abolishes the inhibition of autophagy. MEFs stably expressing GFP-MAP1LC3B were transfected with WT-RNF2 or H69Y-RNF2 mutant, followed by starvation in EBSS for $2 \mathrm{~h}$ with or without BafA1 treatment. GFP-MAP1LC3B dots were counted and are shown in the lower panel. Data are representative of at least three separate experiments and shown as means $\pm \mathrm{SD}$. Scale bar, $10 \mu \mathrm{m}$. ${ }^{* *} P<0.01 ;{ }^{* *} P<0.001$. 
A

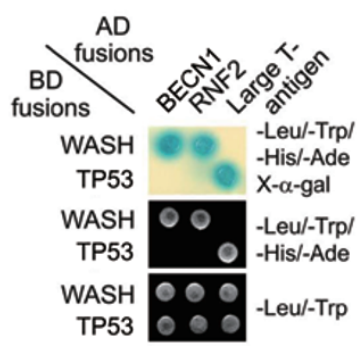

D

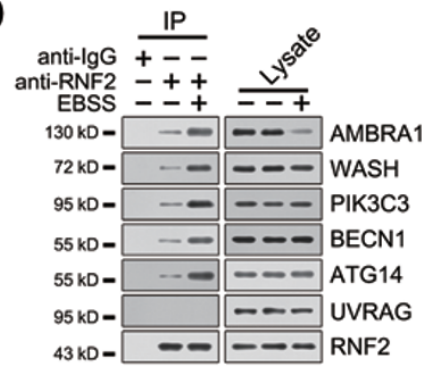

G

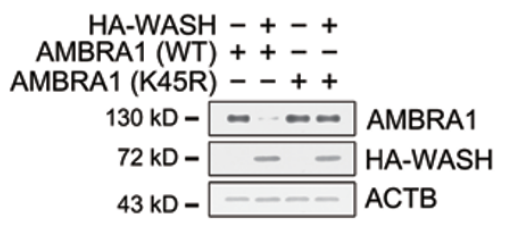

B

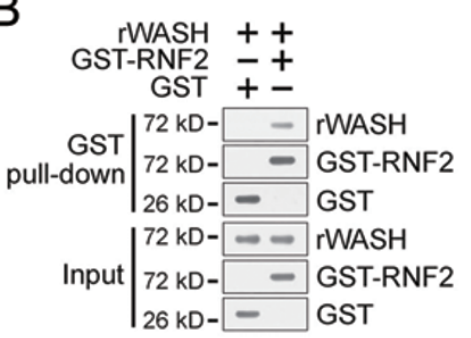

E

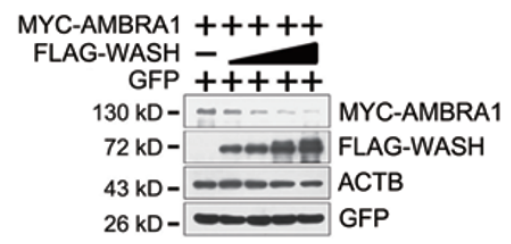

$\mathrm{H}$
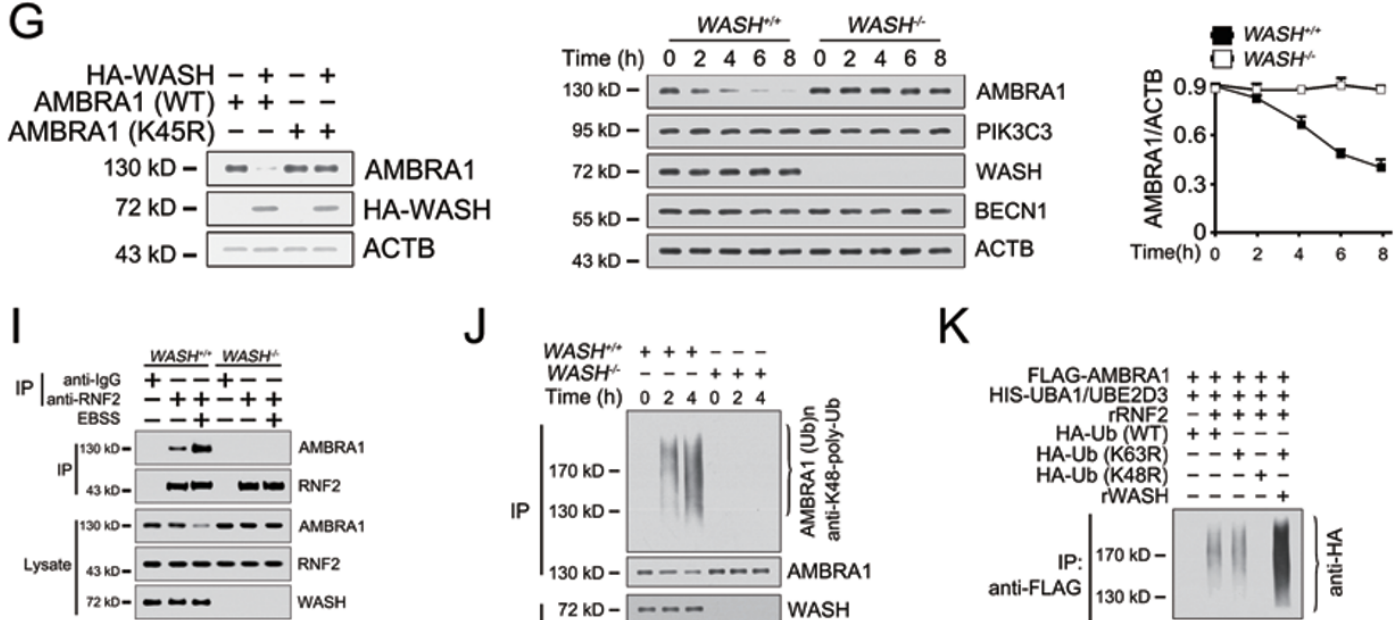

J

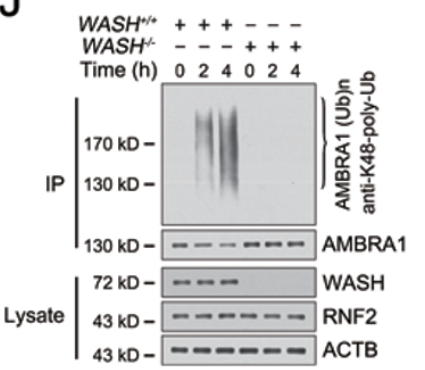

C

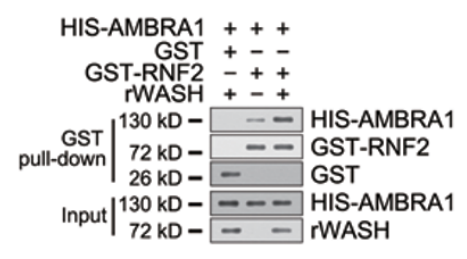

F

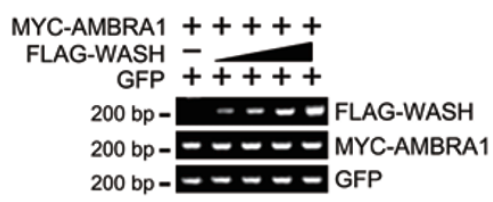

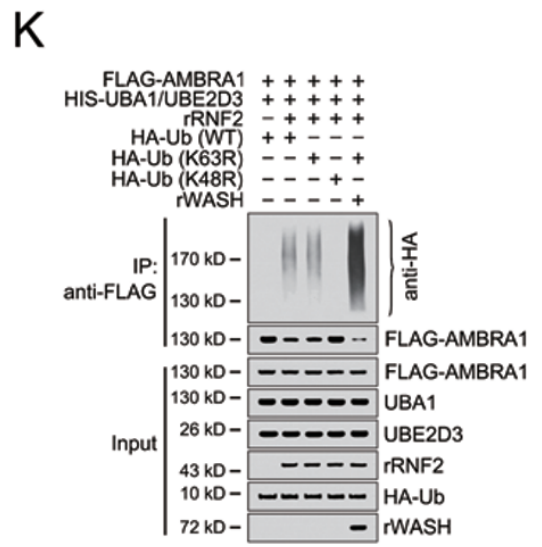

Figure 7 WASH mediates RNF2 recruitment for AMBRA1 degradation. (A) WASH interacts with RNF2 in yeast two-hybrid assays. Yeast strain AH109 was transfected with BD-fused WASH and AD-fused RNF2 and plated on the indicated medium for $3 \mathrm{~d}$. TP53 with large T antigen or a known WASH interactor Bloc1s2 (data not shown) was introduced as positive controls. (B) WASH directly interacts with RNF2. Recombinant WASH (rWASH) and GST-RNF2 were subjected to GST pulldown assays. (C) WASH enhances the interaction between RNF2 and AMBRA1. GST-RNF2 or GST was incubated with recombinant AMBRA1 with or without WASH, followed by GST pull-down assays. (D) RNF2 associates with the components of the BECN1-PIK3C3 complex. Cell lysates from MEFs treated with or without EBSS for $2 \mathrm{~h}$ were immunoprecipitated with anti-RNF2 antibody. (E, F) WASH promotes degradation of AMBRA1. MEFs transfected with MYC-AMBRA1 and increasing amounts of FLAG-WASH were harvested $24 \mathrm{~h}$ post-transfection for immunoblotting with the indicated antibodies (E). mRNAs were extracted and subjected to RT-PCR analysis with primers specific for exogenous plasmids (F). GFP served as a transfection control. (G) K45R-AMBRA1 mutation abolishes WASH-augmented degradation of AMBRA1. HA-tagged WASH was transfected into AMBRA1-depleted MEFs rescued with WT- or K45R-AMBRA1 for $24 \mathrm{~h}$. Cells were starved in EBSS for $4 \mathrm{~h}$, and harvested for immunoblotting with the indicated antibodies. (H) WASH deficiency prevents degradation of AMBRA1. $\mathrm{WASH}^{+/+}$or $\mathrm{WASH}^{--}$MEFs treated with $20 \mu \mathrm{g} / \mathrm{ml} \mathrm{CHX}$ plus EBSS for the indicated times were harvested for immunoblotting (left panel). Blot band intensity ratios of AMBRA1/ACTB were calculated and shown as means \pm SD (right panel). (I) AMBRA1 
in RNF2 KO cells. Expectedly, the number of GFP-WIPI1 dots was significantly increased in RNF2-deficient cells (Figure 6G), and BafA1 treatment further increased GFP-WIPI1 dots, suggesting that RNF2 KO cells exhibited elevated PIK3C3 kinase activity. In addition, we introduced WT-RNF2 and H69Y-RNF2 into MEF cells to examine autophagy induction. We found that WT-RNF2 overexpression abolished autophagy induction (Figure $6 \mathrm{H})$. In contrast, the ligase-dead H69Y-RNF2-transfected cells still underwent autophagy with EBSS stimulation. In addition, we observed that AMBRA1 was associated with RNF2, but not the other major PRC1 complex component BMI1 during autophagy (Supplementary information, Figure S4).

\section{WASH recruits RNF2 for AMBRA1 degradation}

We previously screened a human spleen cDNA library by using WASH as a bait [27]. Besides BECN1, RNF2 was identified as another interactor with WASH (Figure 7A). Bloc1s2, a known interactor of WASH [38], was used as a positive control for the yeast two-hybrid assay (data not shown). The direct interaction of WASH with RNF2 was confirmed by pull-down assays with their recombinant proteins (Figure 7B). In addition, WASH enhanced the interaction of GST-RNF2 with AMBRA1 (Figure 7C). Notably, we observed that RNF2 interacted with the components of the Atg14-associated BECN1PIK3C3 complex (Figure 7D).

We next attempted to test whether WASH can recruit RNF2 for AMBRA1 degradation in autophagy. With EBSS treatment, endogenous WASH was able to recruit much more RNF2 (Figure 7D). We further observed that WASH could attenuate the protein level of AMBRA1 in a dose-dependent manner (Figure 7E), while WASH did not affect the mRNA level of AMBRA1 (Figure 7F).

To further examine whether WASH promotes the degradation of AMBRA1, we overexpressed WT-AMBRA1 or K45R-AMBRA1 mutant with WASH. As shown in Figure 7G, WASH overexpression promoted the degradation of WT-AMBRA1, but not K45R-AMBRA1, suggesting that RNF2-catalyzed AMBRA1 ubiquitination is critical for AMBRA1 degradation. By contrast, WASH deficiency impaired AMBRA1 degradation during autophagy induction (Figure $7 \mathrm{H}$ ). In addition, WASH deficiency did not impair the mRNA level of AMBRA1 (Supplementary information, Figure S5). Notably, anti-RNF2 antibody failed to immunoprecipitate AMBRA1 in $\mathrm{WASH}^{-/}$cells, whereas anti-RNF2 antibody was able to immunoprecipitate AMBRA1 in $W_{A S H}{ }^{+/+}$cells and precipitated much more amount of AMBRA1 in autophagy (Figure 7I). These data indicate that WASH enhances the association of RNF2 with AMBRA1

Of note, WASH deficiency abolished K48-linked polyubiquitination of AMBRA1 during autophagy induction (Figure 7J). To further confirm that WASH mediates K48-linked AMBRA1 ubiquitination, we performed an in vitro AMBRA1 ubiquitination reconstitution assay. WASH indeed promoted RNF2-mediated K48-linked polyubiquitination of AMBRA1 (Figure 7K). In sum, WASH can recruit RNF2 to catalyze K48-linked polyubiquitination of AMBRA1 for its degradation, leading to downregulation of autophagy.

\section{Discussion}

We recently showed that WASH is an inhibitor of autophagy [27]. We identified that WASH is a new interactor of BECN1 to inhibit autophagy through suppression of the ubiquitination of BECN1. The AMBRA1-DDB1-CUL4A complex acts as an E3 ligase for K63-linked ubiquitination of BECN1 that augments PIK3C3 activity. Here, we show that RNF2 negatively regulates autophagy through promoting AMBRA1 degradation. Importantly, we demonstrated that WASH and RNF2 exert inhibitory function in the autophagy induction. RNF2 is firstly identified in the PRC1 complex, which is responsible for monoubiquitination of histone H2A at lysine 119 [31, 39] and polyubiquitination of DNA replication inhibitor Geminin for its degradation [33]. Here, we identified that RNF2 acts as an E3 ligase of AMBRA1 for its polyubiquitination. Upon autophagy induction, WASH mediates recruitment of RNF2 to polyubiquitinate AMBRA1 for its degradation, leading to suppression of autophagy.

AMBRA1, also known as DCAF3, interacts with the DDB1-CUL4A E3 ubiquitin ligase complex [40, 41]. DCAF substrate receptors confer its ligase specificity $[40,42,43]$. AMBRA1 was reported to be a positive regulator of autophagy [13, 24-26]. It was firstly

associates with RNF2 dependently of WASH. WASH ${ }^{+/+}$or $\mathrm{WASH}^{-1-} \mathrm{MEFs}$ were treated with or without EBSS for $2 \mathrm{~h}$, and cell lysates were immunoprecipitated with anti-RNF2 antibody. (J) WASH deficiency impairs polyubiquitination of AMBRA1. $\mathrm{WASH}^{+/+}$or $\mathrm{WASH}^{-/}$MEFs were starved in EBSS for the indicated times, followed by IP with antibody against AMBRA1 after pretreatment with $10 \mu \mathrm{M}$ MG132 for $6 \mathrm{~h}$. Immunoprecipitates were immunoblotted with the indicated antibodies. (K) WASH promotes polyubiquitination of AMBRA1 in vitro. Recombinant RNF2 was incubated with purified FLAG-AMBRA1, E1 (UBA1), E2 (UBE2D3) and ubiquitin mutants with or without recombinant WASH at $30^{\circ} \mathrm{C}$ for $1 \mathrm{~h}$, followed by IP with antibody against FLAG and immunoblotting with the indicated antibodies. Experiments were repeated three times with similar results. 
reported to be an interactor of $\mathrm{BECN} 1$ and that it can enhance kinase activity of PIK3C3 through increasing the association between BECN1 and PIK3C3 [13]. A recent study showed that AMBRA1 tethers the BECN1PIK3C3 complex to cytoskeleton to inhibit autophagy under normal conditions [24]. The same group also reported that AMBRA1 can be phosphorylated at Ser52 by mTOR to impair ULK1 stability and activity, leading to suppression of autophagy in a steady state [25]. Upon autophagy, AMBRA1 undergoes dephosphorylation. Our previous study showed that AMBRA1 acts as a substrate adaptor linking the DDB1-CUL4A ligase complex to BECN1 [27]. The AMBRA1-DDB1-CUL4A ligase complex mediates K63-linked ubiquitination of BECN1, which enhances the association of BECN1 with PIK3C3 during autophagy induction. Here, we found that AMBRA1 is degraded upon autophagy. WASH can recruit RNF2 to ubiquitinate AMBRA1 via K48-linkage for its degradation through a proteasome degradation pathway. We verified that RNF2 serves as a new E3 ligase for K48-linked ubiquitination of AMBRA1. AMBRA1 degradation disrupts the AMBRA1-DDB1-CUL4A E3 ligase complex to block ubiquitination of BECN1. Recent reports showed that CUL4A and TRAF6 associate with AMBRA1 [25, 44, 45]. However, whether CUL4A and TRAF6 are the E3 ligases for AMBRA1 degradation in starvation-induced autophagy needs to be further investigated.

During the induction of autophagy, the isolation membrane (phagophore) elongates and seals itself to produce an autophagosome $[3,12,46]$. The autophagosome fuses with endocytic cargos to generate a mature autophagosome. How this process is regulated remains elusive. Autophagosome formation has been reported to be regulated by at least four complexes, including the ULK1 complex, the BECN1-PIK3C3 complex, the Atg9 and WIPI complex, and the Atg12 and MAP1LC3B conjugation system [3]. Among them, the BECN1-PIK3C3 complex has been extensively studied [47-49]. Up to date, many components have been identified in the BECN1-PIK3C3 complex, including ATG14, UVRAG, AMBRA1, and Rubicon [13, 15, 16, 49]. During autophagy, PI3P is essential for accumulating regulatory factors to the site of autophagosome formation. $\mathrm{PI} 3 \mathrm{KC} 3$ is responsible for the most of PI3P synthesis during autophagy [5, 12]. Similar to its ortholog in yeast, PIK3C3 in mammalian cells exists in two distinct complexes, in which BECN1, PIK3C3 and $\mathrm{p} 150 / \mathrm{Vps} 15$ are the core components [14-16, 47]. The PIK3C3 complexes are finely controlled by each component. UVRAG can activate the BECN1-PIK3C3 complex when cells encounter nutrient limitation [47]. ATG14 (yeast Atg14-like) promotes PIK3C3 kinase activity and enhances autophagy $[15,16]$. Notably, Rubicon is also assembled in the BECN1-PIK3C3 complex, which inhibits autophagy through disturbing autophagosome maturation [16]. Interestingly, we showed that WASH and AMBRA1 are present in the BECN1PIK3C3 complex and colocalize with MAP1LC3B, suggesting that these proteins are involved in the regulation of autophagosome formation [27]. In this study, we observed that RNF2 associates with the BECN1PIK3C3 complex to regulate autophagy. The interaction between BECN1 and PIK3C3 is highly dynamic [50]. In normal conditions, BECN1 is retained in the ER by binding to $\mathrm{Bcl} 2$ [22]. The BECN1 and PIK3C3 interaction is very weak at this moment. Upon autophagy induction, BECN1 dissociates from Bcl2 to bind PIK3C3. We previously found that BECN1 is ubiquitinated by the AMBRA1-DDB1-CUL4A complex through K63-linkage during autophagy [27]. Polyubiquitinated BECN1 has an elevated capacity to bind $\mathrm{PIK} 3 \mathrm{C} 3$, resulting in an enhanced PIK3C3 kinase activity. Here, we found that WASH can recruit RNF2 to ubiquitinate K48-linked AMBRA1 for its degradation, leading to suppression of autophagy.

We previously showed that WASH and AMBRA1 are present in the BECN1-PIK3C3 complex [27]. We demonstrated that WASH overexpression dramatically reduced the association between BECN1 and AMBRA1 [27], which suggests that WASH suppresses the BECN1 ubiquitination through a competitive binding inhibition. However, the competitive binding inhibition did not completely block the engagement of BECN1 to AMBRA1 even with excessive overexpression of WASH [27]. These data imply that an alternative pathway may exist to inhibit the BECN1 ubiquitination in the regulation of autophagy. In this study, we show another novel layer of regulation of autophagy by promoting the degradation of the BECN1 E3 ligase AMBRA1 to impair the BECN1 ubiquitination. Besides the competitive binding inhibition, WASH can also recruit RNF2 to ubiquitinate AMBRA1 via K48-linkage, leading to AMBRA1 degradation. These two regulatory layers ensure that K63-linked ubiquitination of BECN1 is completely blocked in order to terminate autophagy.

Combining our present and previous findings, we suggest the following model. For autophagy induction, BECN1 is rapidly ubiquitinated via K63-linkage by the AMBRA1-DDB1-CUL4A E3 ligase complex, and then ubiquitinated BECN1 binds to PIK3C3 to augment PIK3C3 kinase activity. For autophagy suppression, WASH recruits the E3 ligase RNF2 that ubiquitinates AMBRA1 via K48-linkage that leads to AMBRA1 degradation. AMBRA1 degradation or competitive bind- 
ing inhibition impedes BECN1 ubiquitination. Thus, non-ubiquitinated BECN1 fails to associate with PIK3C3 and activate its kinase activity, leading to autophagy suppression.

\section{Materials and Methods}

\section{Antibodies and reagents}

Anti-MAP1LC3B (3868), anti-BECN1 (3738), anti-RNF2 (5694), anti-AMBRA1 (12250), anti-poly-Ub (3936), anti-K63Ub (5621), anti-BMI1 (6964), and anti-PIK3C3 (3358) were from Cell Signaling Technology. Mouse monoclonal antibodies anti-ACTB (A5441), anti-HIS (P1967), anti-HA (H3663), anti-GST (SAB5300159), and anti-FLAG (F3040) were from Sigma-Aldrich. Rabbit polyclonal antibodies anti-MAP1LC3B (PM036) and anti-SQSTM1 (PM045) were from MBL. Rabbit polyclonal antibody anti-HA (sc-805) and mouse monoclonal antibody anti-MYC (sc-40) were from Santa Cruz Biotechnology. Donkey anti-rabbit IgG secondary antibodies conjugated with Alexa-488 (A11008), Alexa-594 (A11012) or Alexa-405 (A31553) were purchased from Molecular Probes, Invitrogen. Donkey anti-mouse IgG secondary antibodies conjugated with Alexa-488 (A11029) or Alexa-594 (A11005) were from Molecular Probes, Invitrogen. HRP-conjugated secondary antibodies were from Santa Cruz Biotechnology. BafA1 (B1793), cycloheximide (CHX; 01810), Staurosporine (S6942), Z-VAD (V116), and MG132 (M7449) were from Sigma-Aldrich.

\section{Generation of $\mathrm{WASH}^{--}$and $\mathrm{RNF}^{-/-} \mathrm{MEFS}$}

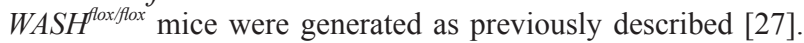
$R N F 2^{f l o x} f f o x$ mice were generously provided by Dr Haruhiko Koseki (RIKEN, Japan). For $R N F 2^{f l o x f l o x}$ mouse generation, the transcription start site of RinglB was flanked by loxP sites, followed by insertion of the PGKneo selection marker into the first intron [51]. Conditional KO mice were produced through a conventional KO breading scheme. MEFs were prepared from E14.5 embryos and infected with lentivirus encoding the Cre recombinase for $48 \mathrm{~h}$. MEF cells were further selected with $2 \mu \mathrm{g} / \mathrm{ml}$ puromycin to generate WASH- and RNF2-deficient cells. WASH and RNF2 expressions were confirmed by western blotting and immunostaining.

\section{Yeast two-hybrid screening}

Yeast two-hybrid screening was conducted following guideline provided by the manufacturer (Matchmaker Gold Yeast Two-Hybrid system, Clontech/Takara). Briefly, WASH and RNF2 were cloned into pGBKT7 vector, respectively. Yeast strain AH109 cells were transfected with pGBKT7-RNF2 or pGBKT7-WASH and plasmids containing a human spleen cDNA library (Clontech/ Takara), followed by selection on SD medium without adenine, histidine, tryptophan, and leucine as described [27]. Clones were selected and identified by DNA sequencing.

\section{Autophagy induction by starvation}

MEFs were cultured in Dulbecco's modified Eagle's medium (Gibco, 11965118), containing 10\% fetal bovine serum (Gibco, 10091148), $\beta$-mercaptoethanol (Sigma-Aldrich, M6250), $100 \mu \mathrm{g} /$ $\mathrm{ml}$ streptomycin (Sigma-Aldrich, S6501) and $100 \mathrm{U} / \mathrm{ml}$ penicillin (Sigma-Aldrich, 13752). EBSS (Invitrogen, 14155063) was used to as starvation culture for autophagy induction.

\section{Immunofluorescence}

MEFs were grown on $0.01 \%$ poly-L-Lysine-treated coverslips and transfected with the indicated vectors by Lipofectamine 2000 (Invitrogen, 11668019) for $24 \mathrm{~h}$ as described [52]. After starvation stimulation, cells were fixed with $4 \%$ paraformaldehyde (Sigma-Aldrich, 158127) for $30 \mathrm{~min}$ at RT, followed by permeabilization with $50 \mu \mathrm{g} / \mathrm{ml}$ digitonin for $20 \mathrm{~min}$ at RT. $10 \%$ donkey serum was used for blocking and primary antibodies were added to incubate for $2 \mathrm{~h}$ at RT. After washing with PBS, the coverslips were stained with Alexa488-, Alexa594- or Alexa405-conjugated secondary antibodies. Images were obtained with laser scanning confocal microscopy (Olympus, FV1200). For calculation of dots/ puncta, MAP1LC3B or ATG5 dots with diameter $>0.5 \mu \mathrm{m}$ were counted and at least 100 cells were examined for each experiment.

\section{$R N A$ interference}

RNA interference sequences were designed according to pSUPER system instructions (Oligoengine). MEFs were electroporated with pSUPER vector encoding target sequences against AMBRA1 (\#1: 5'-CAGTGAGAACAACTCCAAC-3', \#2: 5'-TGGTGAAGACAGCTAGTGA-3'), BMI1 (\#1: 5'-AGCAGATTGGATCGGAAAG-3', \#2: 5'-CGCTAATGGACATTGCCTA-3'), and scramble sequences. $48 \mathrm{~h}$ after electroporation, stably silenced clones were selected by $1 \mu \mathrm{g} / \mathrm{ml}$ puromycin.

\section{Electron microscopy}

For transmission electron microscopy, MEFs were harvested by trypsin digestion and fixed with $2.5 \%$ glutaraldehyde on ice for 2 $\mathrm{h}$ followed by postfixation in $2 \%$ osmium tetroxide [53]. Briefly, cells were immersed in SPI-PON812 resin after dehydration with sequential washes in $50 \%, 70 \%, 90 \%, 95 \%$, and $100 \%$ ethanol. The ultrathin sections were collected on copper grids and counterstained using uranyl acetate and lead citrate. For electron microscopy of mouse embryos, E8.5 embryos were prepared and treated the same as above. Images were taken with a FEI Tecnai spirit transmission electron microscope.

\section{In vitro ubiquitination reconstitution assay}

UBA1, UBE2D3, and ubiquitin were subcloned into pET28 a vectors. RNF2 and AMBRA1 were subcloned into pMAL$\mathrm{c} 2 \mathrm{p}$ vectors. Plasmids were then transformed into $E$. coli strain BL21 (DE3). DE3 clones were cultured (OD600 = 0.6) and then induced with $0.2 \mathrm{mM}$ isopropyl- $\beta$-d-thiogalactopyranoside (Sigma-Aldrich, E005502) at $16{ }^{\circ} \mathrm{C}$ for $24 \mathrm{~h}$. Cells were collected and lysed by supersonic. The lysate was further purified by Ni-NTA resin columns, MBP columns (New England Biolabs, E8021S) or GST-sepharose columns (GE-Healthcare, 17-0756-01). The in vitro ubiquitination reconstruction assays were performed by mixing E1 (UBA1), E2 (UBE2D3), E3 (RNF2), ubiquitin, and FLAG-AMBRA1 in the ubiquitination buffer ( $50 \mathrm{mM}$ Tris- $\mathrm{HCl}, 5$ $\mathrm{mM} \mathrm{MgCl} 2,2 \mathrm{mM}$ dithiothreitol, and $2 \mathrm{mM}$ ATP, $\mathrm{pH}$ 7.4) of $30 \mu \mathrm{l}$ volume at $37^{\circ} \mathrm{C}$ for $2 \mathrm{~h}$.

\section{Immunoprecipitation}

MEFs were electroporated with the indicated vectors for $24 \mathrm{~h}$. Cells were harvested and treated with lysis buffer $(150 \mathrm{mM} \mathrm{NaCl}$, $50 \mathrm{mM}$ Tris- $\mathrm{HCl}, 1 \%$ TritonX-100, protease inhibitor cocktail, $\mathrm{pH}$ 7.4). Supernatants were achieved by centrifugation $(15000 \times g, 15$ min, $4{ }^{\circ} \mathrm{C}$ ), and incubated with the indicated antibodies for $6 \mathrm{~h}$ at $4{ }^{\circ} \mathrm{C}$ followed by IP with $30 \mu 1$ protein $\mathrm{A} / \mathrm{G}$ agarose (Santa Cruz 
Biotechnology, sc-2003). The precipitates were completely washed with PBS and tested by immunoblotting. To detect polyubiquitinated AMBRA1, immunoprecipitates were denatured by boiling with $1 \%$ SDS and reimmunoprecipitated with anti-AMBRA1 antibody.

\section{PIK3C3 kinase assay}

After being treated with culture medium or EBSS (starvation) for $1 \mathrm{~h}$, MEFs transfected with the indicated plasmids were harvested. Cells were lysed in lysis buffer $(150 \mathrm{mM} \mathrm{NaCl}, 50 \mathrm{mM}$ Tris-HCl, 1\% TritonX-100, protease inhibitor cocktail, $\mathrm{pH} 7.4$ ) and immunoprecipitation with anti-FLAG antibody was performed as described above. Immunoprecipitates were split into two equal parts, one for a loading control and the other for in vitro kinase assay. Immunoprecipitates were washed three times in washing buffer containing $50 \mathrm{mM}$ Tris- $\mathrm{HCl}$ and $500 \mathrm{mM} \mathrm{LiCl}, \mathrm{pH} \mathrm{7.4,}$ followed by washing for three times in a reaction buffer containing $10 \mathrm{mM}$ Tris- $\mathrm{HCl}, 100 \mathrm{mM} \mathrm{NaCl}$ and $1 \mathrm{mM}$ EDTA. Immunoprecipitates were then resuspended in $50 \mu 1$ reaction buffer and $15 \mathrm{mM} \mathrm{MnCl}_{2}$ before $20 \mu \mathrm{g}$ sonicated phosphatidylinositol was added. The reaction was performed in the presence of $50 \mu \mathrm{M}$ ATP and stopped by the addition of $1.5 \mathrm{M} \mathrm{HCl}$. Organic phase was extracted with $200 \mu \mathrm{l}$ chloroform:methanol (v/v, 1:1) and resolved by TLC using a coated silica gel in a solvent comprising chloroform:methanol: $\mathrm{H}_{2} \mathrm{O}:$ ammonium hydroxide (v/v/v/v, 9:7:1.7:0.3). The gel was imaged by Typhoon 9400 Variable Imager (GE Healthcare, Typhoon 9400).

\section{Statistical analysis}

Student's $t$-test was used as statistical analysis by Microsoft Excel.

\section{Acknowledgments}

We thank Dr Haruhiko Koseki (RIKEN) for providing RNF$2^{f l o x f f o x}$ mice. We thank Xuan Yang for protein expression and Yan Teng for technical support. We also thank Drs Liang Tong and Dangsheng Li for critical reading and suggestions. This work was supported by the National Natural Science Foundation of China (31300645, 81330047, 30830030, 30972676), 973 Program of the MOST of China (2010CB911902), the Strategic Priority Research Programs of the Chinese Academy of Sciences (XDA01010407).

\section{References}

1 Bodemann BO, Orvedahl A, Cheng TL, et al. RalB and the exocyst mediate the cellular starvation response by direct activation of autophagosome assembly. Cell 2011; 144:253-267.

2 Lee JH, Yu WH, Kumar A, et al. Lysosomal proteolysis and autophagy require presenilin 1 and are disrupted by Alzheimer-related PS1 mutations. Cell 2010; 141:1146-1158.

3 Mizushima N, Komatsu M. Autophagy: renovation of cells and tissues. Cell 2011; 147:728-741.

4 Shintani T, Klionsky DJ. Autophagy in health and disease: A double-edged sword. Science 2004; 306:990-995.

5 Axe EL, Walker SA, Manifava M, et al. Autophagosome formation from membrane compartments enriched in phosphatidylinositol 3-phosphate and dynamically connected to the endoplasmic reticulum. $J$ Cell Biol 2008; 182:685-701.

6 Hailey DW, Rambold AS, Satpute-Krishnan P, et al. Mito- chondria supply membranes for autophagosome biogenesis during starvation. Cell 2010; 141:656-667.

7 Hayashi-Nishino M, Fujita N, Noda T, Yamaguchi A, Yoshimori T, Yamamoto A. A subdomain of the endoplasmic reticulum forms a cradle for autophagosome formation. Nat Cell Biol 2009; 11:1433-1437.

8 Reggiori F. Membrane origin for autophagy. Curr Top Dev Biol 2006; 74:1-30.

9 Yla-Anttila P, Vihinen H, Jokita E, Eskelinen EL. 3D tomography reveals connections between the phagophore and endoplasmic reticulum. Autophagy 2009; 5:1180-1185.

10 Liang CY, Lee JS, Inn KS, et al. Beclin1-binding UVRAG targets the class $\mathrm{C}$ Vps complex to coordinate autophagosome maturation and endocytic trafficking. Nat Cell Biol 2008; 10:776-787.

11 Kang R, Zeh HJ, Lotze MT, Tang D. The Beclin 1 network regulates autophagy and apoptosis. Cell Death Differ 2011; 18:571-580.

12 Weidberg H, Shvets E, Elazar Z. Biogenesis and cargo selectivity of autophagosomes. Annu Rev Biochem 2011; 80:125156.

13 Fimia GM, Stoykova A, Romagnoli A, et al. Ambra1 regulates autophagy and development of the nervous system. $\mathrm{Na}$ ture 2007; 447:1121-1125.

14 Itakura E, Kishi C, Inoue K, Mizushima N. Beclin 1 forms two distinct phosphatidylinositol 3-kinase complexes with mammalian Atg14 and UVRAG. Mol Biol Cell 2008; 19:5360-5372.

15 Matsunaga K, Saitoh T, Tabata K, et al. Two Beclin 1-binding proteins, Atg14L and Rubicon, reciprocally regulate autophagy at different stages. Nat Cell Biol 2009; 11:385-396.

16 Zhong Y, Wang QJ, Li XT, et al. Distinct regulation of autophagic activity by Atg14L and Rubicon associated with Beclin 1-phosphatidylinositol-3-kinase complex. Nat Cell Biol 2009; 11:468-476.

17 Aita VM, Liang XH, Murty VVVS, et al. Cloning and genomic organization of beclin 1 , a candidate tumor suppressor gene on chromosome 17q21. Genomics 1999; 59:59-65.

18 Lindmo K, Stenmark H. Regulation of membrane traffic by phosphoinositide 3-kinases. J Cell Sci 2006; 119:605-614.

19 Simonsen A, Tooze SA. Coordination of membrane events during autophagy by multiple class III PI3-kinase complexes. J Cell Biol 2009; 186:773-782.

20 Yan Y, Flinn RJ, Wu HY, Schnur RS, Backer JM. hVps15, but not $\mathrm{Ca} 2+/ \mathrm{CaM}$, is required for the activity and regulation of hVps34 in mammalian cells. Biochem J 2009; 417:747-755.

$21 \mathrm{Ku} \mathrm{B}$, Woo JS, Liang C, Lee KH, Jung JU, Oh BH. An insight into the mechanistic role of Beclin 1 and its inhibition by prosurvival Bcl-2 family proteins. Autophagy 2008; 4:519-520.

22 Pattingre S, Tassa A, Qu XP, et al. Bcl-2 antiapoptotic proteins inhibit Beclin 1-dependent autophagy. Cell 2005; 122:927-939.

23 Wei YJ, Pattingre S, Sinha S, Bassik M, Levine B. JNK1-mediated phosphorylation of BcI-2 regulates starvation-induced autophagy. Mol Cell 2008; 30:678-688.

24 Di Bartolomeo S, Corazzari M, Nazio F, et al. The dynamic interaction of AMBRA1 with the dynein motor complex regulates mammalian autophagy. J Cell Biol 2010; 191:155-168.

25 Nazio F, Strappazzon F, Antonioli M, et al. mTOR inhibits 
autophagy by controlling ULK1 ubiquitylation, self-association and function through AMBRA1 and TRAF6. Nat Cell Biol 2013; 15:406-416.

26 Strappazzon F, Vietri-Rudan M, Campello S, et al. Mitochondrial BCL-2 inhibits AMBRA1-induced autophagy. EMBO J 2011; 30:1195-1208.

27 Xia P, Wang S, Du Y, et al. WASH inhibits autophagy through suppression of Beclin 1 ubiquitination. EMBO J 2013; 32:2685-2696.

28 Levine SS, Weiss A, Erdjument-Bromage H, Shao ZH, Tempst P, Kingston RE. The core of the polycomb repressive complex is compositionally and functionally conserved in flies and humans. Mol Cell Biol 2002; 22:6070-6078.

29 Pirrotta V, Poux S, Melfi R, Pilyugin M. Assembly of polycomb complexes and silencing mechanisms. Genetica 2003; 117:191-197.

30 Buchwald G, van der Stoop P, Weichenrieder O, Perrakis A, van Lohuizen M, Sixma TK. Structure and E3-ligase activity of the Ring-Ring complex of polycomb proteins Bmil and Ring1b. EMBO J 2006; 25:2465-2474.

31 Wang HB, Wang LJ, Erdjument-Bromage H, et al. Role of histone $\mathrm{H} 2 \mathrm{~A}$ ubiquitination in polycomb silencing. Nature 2004; 431:873-878.

32 Voncken JW, Roelen BAJ, Roefs M, et al. Rnf2 (Ring1b) deficiency causes gastrulation arrest and cell cycle inhibition. Proc Nat Acad Sci USA 2003; 100:2468-2473.

33 Ohtsubo M, Yasunaga S, Ohno Y, et al. Polycomb-group complex 1 acts as an E3 ubiquitin ligase for Geminin to sustain hematopoietic stem cell activity. Proc Nat Acad Sci USA 2008; 105:10396-10401.

34 Su WJ, Fang JS, Cheng F, Liu C, Zhou F, Zhang J. RNF2/ Ring $1 b$ negatively regulates p53 expression in selective cancer cell types to promote tumor development. Proc Nat Acad Sci USA 2013; 110:1720-1725.

35 Lee SJ, Choi D, Rhim H, Kang S. E3 ubiquitin ligase RNF2 interacts with the S6' proteasomal ATPase subunit and increases the ATP hydrolysis activity of S6'. Biochem J 2005; 389:457-463.

36 Ciechanover A. Proteolysis: from the lysosome to ubiquitin and the proteasome. Nat Rev Mol Cell Biol 2005; 6:79-86.

37 Proikas-Cezanne T, Ruckerbauer S, Stierhof YD, Berg C, Nordheim A. Human WIPI-1 puncta-formation: A novel assay to assess mammalian autophagy. FEBS Lett 2007; 581:33963404.

38 Monfregola J, Napolitano G, D’Urso M, Lappalainen P, Ursini MV. Functional characterization of Wiskott-Aldrich syndrome protein and scar homolog (WASH), a bi-modular nucleation-promoting factor able to interact with biogenesis of lysosome-related organelle subunit 2 (BLOS2) and gamma-tubulin. J Biol Chem 2010; 285:16951-16957.

39 de Napoles M, Mermoud JE, Wakao R, et al. Polycomb group proteins Ring1A/B link ubiquitylation of histone H2A to heritable gene silencing and X inactivation. Dev Cell 2004; 7:663-676.

40 Fischer ES, Scrima A, Bohm K, et al. The molecular basis of
CRL4DDB2/CSA ubiquitin ligase architecture, targeting, and activation. Cell 2011; 147:1024-1039.

41 Jin J, Arias EE, Chen J, Harper JW, Walter JC. A family of diverse Cul4-Ddb1-interacting proteins includes Cdt2, which is required for $\mathrm{S}$ phase destruction of the replication factor $\mathrm{Cdt}$. Mol Cell 2006; 23:709-721.

42 Bennett EJ, Rush J, Gygi SP, Harper JW. Dynamics of cullin-RING ubiquitin ligase network revealed by systematic quantitative proteomics. Cell 2010; 143:951-965.

43 Wertz IE, O'Rourke KM, Zhang Z, et al. Human De-etiolated-1 regulates c-Jun by assembling a CUL4A ubiquitin ligase. Science 2004; 303:1371-1374.

44 Kim W, Bennett EJ, Huttlin EL, et al. Systematic and quantitative assessment of the ubiquitin-modified proteome. Mol Cell 2011; 44:325-340.

45 Wagner SA, Beli P, Weinert BT, et al. Proteomic analyses reveal divergent ubiquitylation site patterns in murine tissues. Mol Cell Proteomics 2012; 11:1578-1585.

46 Juhasz G, Neufeld TP. Autophagy: A forty-year search for a missing membrane source. PLoS Biol 2006; 4:161-164.

47 Liang C, Feng P, Ku B, et al. Autophagic and tumour suppressor activity of a novel Beclin1-binding protein UVRAG. Nat Cell Biol 2006; 8:688-699.

48 Liu JL, Xia HG, Kim M, et al. Beclin1 controls the levels of p53 by regulating the deubiquitination activity of USP10 and USP13. Cell 2011; 147:223-234.

49 Takahashi Y, Coppola D, Matsushita N, et al. Bif-1 interacts with Beclin 1 through UVRAG and regulates autophagy and tumorigenesis. Nat Cell Biol 2007; 9:1142-1151.

50 Noble CG, Dong JM, Manser E, Song HW. Bcl-x(L) and UVRAG cause a monomer-dimer switch in Beclin1. $J$ Biol Chem 2008; 283:26274-26282.

51 Cales C, Roman-Trufero M, Pavon L, et al. Inactivation of the polycomb group protein Ring1B unveils an antiproliferative role in hematopoietic cell expansion and cooperation with tumorigenesis associated with Ink4a deletion. Mol Cell Biol 2008; 28:1018-1028.

52 Fan ZS, Beresford PJ, Oh DY, Zhang D, Lieberman J. Tumor suppressor NM23-H1 is a granzyme A-activated DNase during CTL-mediated apoptosis, and the nucleosome assembly protein SET is its inhibitor. Cell 2003; 112:659-672.

53 Wang S, Xia P, Ye B, Huang G, Liu J, Fan Z. Transient activation of autophagy via Sox2-mediated suppression of mTOR is an important early step in reprogramming to pluripotency. Cell Stem Cell 2013; 13:617-625.

(Supplementary information is linked to the online version of the paper on the Cell Research website.)

(c) (5) $(-)$ This work is licensed under the Creative Commons Attribution-NonCommercial-No Derivative Works 3.0 Unported License. To view a copy of this license, visit http:// creativecommons.org/licenses/by-nc-nd/3.0 